\title{
MECHANICAL MEASUREMENTS
}

2.1 Introduction to the Field of Biomechanics ..........................................II-1

2.2 Principles Behind Measurements..................................................... II-3

2.3 Techniques in the Field ................................................................. II-5

2.4 Strengths and Limitations of Alternative Techniques:

Illustration in Cornea and Sclera..................................................II-9

2.4.1 Uniaxial Tensile Tests ...............................................................II-10

2.4.2 Oscillatory Shear Rheology .......................................................II-21

2.4.3 Intact Globe Expansion Test ........................................................II-35

Bibliography …………................................................................ II-46

This work includes contributions from Joyce Huynh, Muzhou Wang, and Meredith Wiseman. Undergraduates Muzhou Wang and Meredith Wiseman contributed to the method development necessary for tensile tests and intact globe expansion, respectively. Graduate student Joyce Huynh assisted with intact globe expansion experiments during her first year.

\subsection{Introduction to the Field of Biomechanics}

The field of biomechanics has arisen from a desire to understand how living things work. One of the earliest texts relating to biomechanics came from Aristotle's writing "On the Parts of Animals" (394-322 B.C.). Other contributors to the field include Galileo Galilei, Robert Hooke, Isaac Newton, and Thomas Young. Contributions from them and many others help us understand things ranging from blood flow and respiration to locomotion and talking. 
Findings related to the human body have greatly advanced medicine. Respirators assist with breathing, artificial heart valves replace faulty ones, and bones and joints are replaced with prosthetic devices. Advances within medicine have relied on methodology of engineering and biomechanics to understand problems associated with diseased, injured, or broken systems in relation to healthy working systems. Characterization of bones has lead to earlier detection of osteoporosis, better joint materials, and even injectable materials to fill the holes and gaps in damaged bones.

In relation to the eye, there are obvious mechanical changes of the sclera and cornea associated with degenerative myopia and keratoconus (Chapters 1, 5, \& 6). Characterization of these changes could be useful for understanding and diagnosing the diseases. Understanding the changes that occur could also lead to new insights for treating or preventing the diseases. In addition, clinical treatments aimed at modifying the mechanical properties of the cornea and sclera can be evaluated using methods of biomechanics.

In this chapter we present methods used for the characterization of the cornea and sclera, and we evaluate the reliability of the methods based on the variability of results and the relationship to in vivo conditions. Our findings indicate that results are often methoddependent, and for studies related to keratoconus and degenerative myopia, there are advantages in using an intact globe method that maintains an almost natural configuration of the tissue. 


\subsection{Principles Behind Measurements}

In order to understand the tissue, we are interested in studying the forces imposed on and the mechanical responses of the system. Although biomaterials are typically complex inhomogeneous materials, our understanding of them has been built up from very basic understandings of ideal solids and liquids.

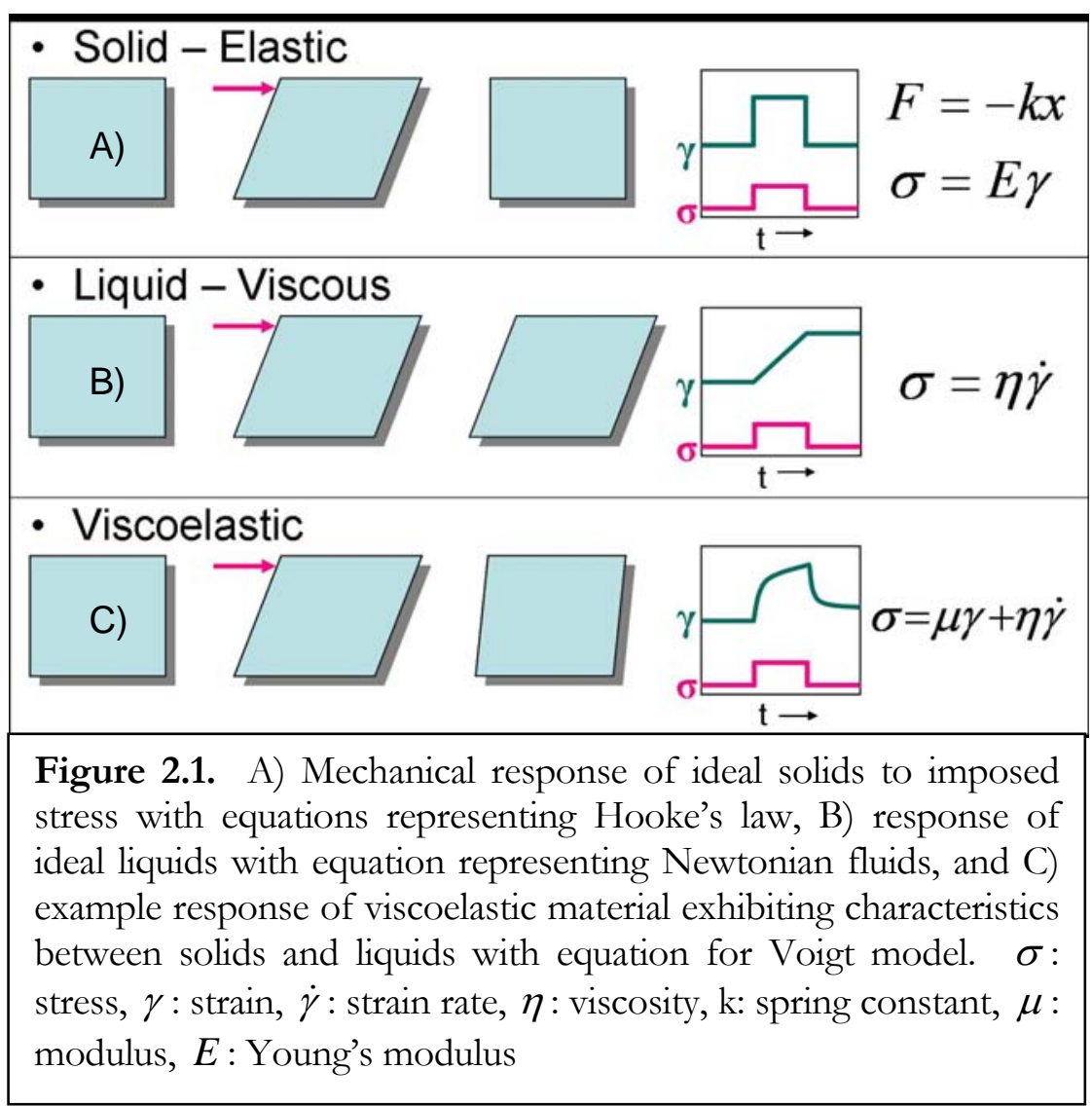

Ideal or Hookian solids can be thought of as isotropic homogeneous materials that behave like springs. Applying a force to the solid induces a deformation. Upon removal of the force, the solid returns to its original shape (Figure 2.1a). This behavior can be described by the restoring force of the spring $F=-k x$. The restoring force $(F)$ is in the opposite 
direction of the imposed deformation $(\mathrm{x})$ and the magnitude of the force depends on material properties of the spring, which are defined by the spring constant $(\mathrm{k})$. Because samples are often different sizes, it is essential to look at the forces relative to sample size in order to isolate the material properties. For this, we examine the stress ( $\sigma$, force per unit cross-sectional area) that is proportional to strain $(\gamma$, a dimensionless measure of deformation). Higher strains create higher stress in the material, or application of higher stresses creates larger strains. The proportionality of stress and strain contains information regarding the material properties and is named Young's modulus, or the elastic modulus (E).

For ideal or Newtonian liquids, it is helpful to think of water flowing through a pipe. If you apply a force to it, water flows along the pipe. If you stop applying a force, then flow stops. There is no restoring force causing the water to return through the pipe (Figure 2.1b). In this case, the amount of deformation is dependent on how long the force is applied; the force is proportional to the deformation rate. As we increase the stress on the liquid, it deforms at a higher strain rate $(\dot{\gamma})$, or if we increase the strain rate, we increase the stress in the liquid. The material properties governing this relation are defined in $\eta$, which is the viscosity of the liquid. Under the same stress, a more viscous liquid (honey) flows slower than a less viscous liquid (water).

Most materials cannot be accurately described by either the ideal solid or ideal liquid, but are more appropriately described by a combination of these. Silly Putty ${ }^{\mathrm{TM}}$ demonstrates the combination of elastic and viscous properties. If you throw a ball of Silly Putty against the floor it will bounce up retaining its normal shape. During the interaction with the floor, it 
acts like a solid, deforming as it hits, but using a restoring force to bounce back. If you slowly pull on Silly Putty it will stretch and elongate. When you stop pulling, the Silly Putty has flowed or creeped and will not return to its original shape. Materials such as Silly Putty, which behave like solids under some conditions and liquids under others, are classified as viscoelastic.

The cornea and sclera are viscoelastic materials that typically exhibit elastic behavior while under physiological stresses. However, under normal intraocular pressures, the cornea and sclera creep in keratoconus and degenerative myopia. This creep is what results in the corneal bulging and axial elongation characteristic of the diseases. Characterization of the elastic and viscous properties of the tissues is essential for understanding the diseases and developing appropriate treatments.

\subsection{Techniques in the Field}

The ability to characterize the mechanical properties of biomaterials lies in the ability to make measurements of the material response to stress and strain. Typically, this is done by applying one (e.g., stress) and recording the other (e.g., strain or strain rate). The way in which this is done depends in large part on the type of behavior that is being analyzed. The bones and spinal column bear compressive forces due to the weight of the body, tendons are subjected to cyclic tensile stresses, the skin is stretched over the body, blood vessels expand and contract with the pumping of blood, and bones shear past one another in the joints. Because of the variety of forces and deformations to study, there are many 
techniques used in biomechanics (Figure 2.2). Also, some testing techniques are developed specifically to replicate the in vivo conditions.

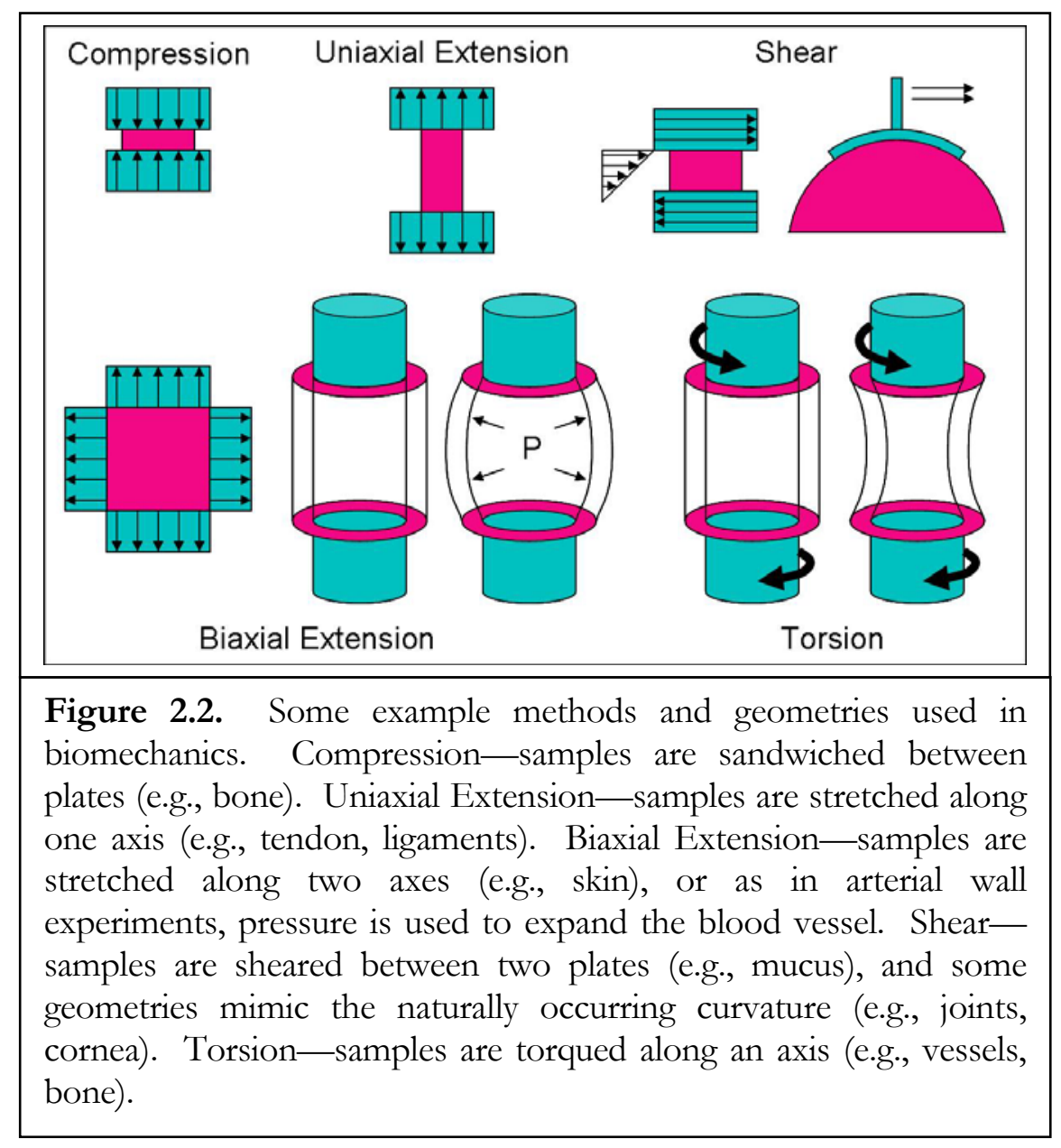

In vivo, the eye is subject to an intraocular pressure that keeps the cornea and sclera stressed. In order to relate this natural state of the eye to types of mechanical testing we can do, it is useful to introduce the Young-Laplace equation (Figure 2.3). The YoungLaplace equation describes the surface tension $(\gamma)$ of a membrane that experiences a pressure differential $(\Delta \mathrm{P})$ across the two sides of the membrane. The curvature of the membrane at any point can be described by the radius of curvature $\left(\mathrm{R}_{\mathrm{x}}\right.$ or $\left.\mathrm{y}\right)$ along the $\mathrm{x}$ - or 
y-axis. By approximating the eye as a sphere $\left(R_{x}=R_{y}=R_{s}\right)$, we are able to simplify this expression to relate the stress in the cornea and sclera to the intraocular pressure (IOP), knowing the radius and thickness of the eye. In a human eye with intraocular pressures of 18-25 mmHg, radius $\sim 12.5 \mathrm{~mm}$, and thickness $\sim 1 \mathrm{~mm}$ the biaxial stress would be $15-21$ KPa. When considering the strip geometry used in uniaxial extension, people often convert this to a tensile force determined by the stress and cross-sectional area of the sample. Samples with a width of $\sim 5 \mathrm{~mm}$ and a thickness of $\sim 1 \mathrm{~mm}$ have a $5 \mathrm{~mm}^{2}$ cross-sectional area, giving a tensile load of $0.075-0.10 \mathrm{~N}$.

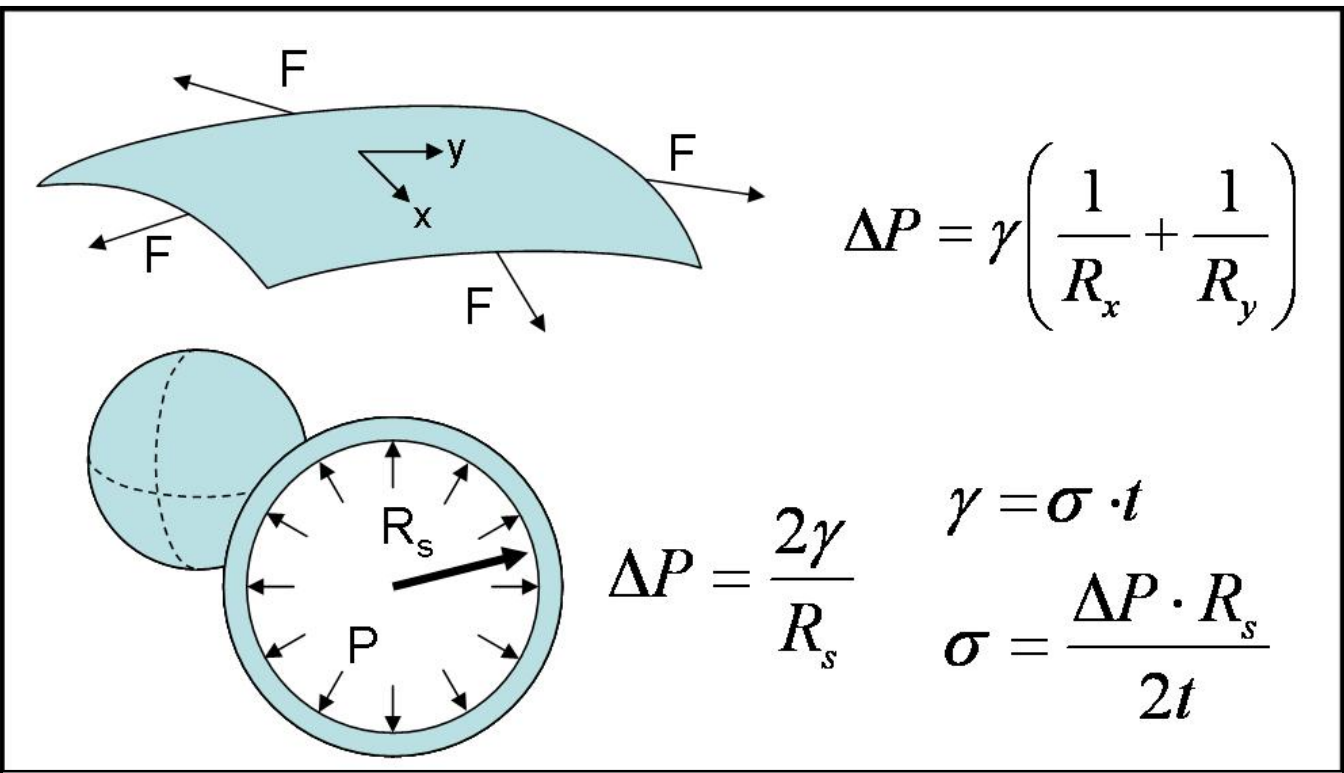

Figure 2.3. The Young-Laplace equation to describe the pressure differential $(\Delta \mathrm{P})$ across a curved membrane with surface tension $(\gamma)$ can be used to approximate the stresses that occur in an eye. By assuming the eye is a spherical shell with $\left(\mathrm{R}_{\mathrm{x}}=\mathrm{R}_{\mathrm{y}}=\mathrm{R}_{\mathrm{s}}\right)$, the relation of intraocular pressure $(\Delta \mathrm{P})$, radius $\left(\mathrm{R}_{\mathrm{s}}\right)$, and thickness $(\mathrm{t})$ can be used to determine stresses $(\sigma)$. This relation becomes useful for the comparison of biomechanical testing methods. 
Cutting tissue samples for biomechanical testing removes the intraocular pressure and natural stress from the specimen. Groups often condition samples to restore the specimen to a state similar to in vivo. For example, application of a pre-stress equivalent to that calculated using the Young-Laplace equation is used as a physiological starting point for measurements. A load (e.g., $\sim 0.1 \mathrm{~N}$ ) is applied for a specified time and the tissue extends under this stress. Unfortunately, the stress distribution in uniaxial tension (where this method is often used) is different than that in biaxial tension and cannot properly simulate in vivo conditions. Another method of conditioning the specimens is the application of cyclic stresses to realign molecules within the sample, and in tensile tests it is often repeated until the hysteresis in the stress-strain curves disappears. Removal of hysteresis implies that the stresses are large enough to cause structural changes in the specimen.

While the application of cyclic stresses is used to precondition tissue, application of small oscillatory stresses can be used to probe the tissue without generating structural changes. Small deformation oscillatory shear rheology oscillates the tissue around zero stress and zero deformation (Figure 2.4). The components of the measured shear stress and strain that are in phase contribute to the magnitude of the storage modulus $\left(\mathrm{G}^{\prime}\right)$, which is related to the elastic properties of the tissue. The components that are out of phase contribute to the magnitude of the loss modulus $\left(\mathrm{G}^{\prime \prime}\right)$, which is related to the viscous properties of the tissue. If the stress and strain were large, it would be possible to monitor the structural changes of the specimen, however the use of small stresses and deformations allows gentle probing of the tissue. 


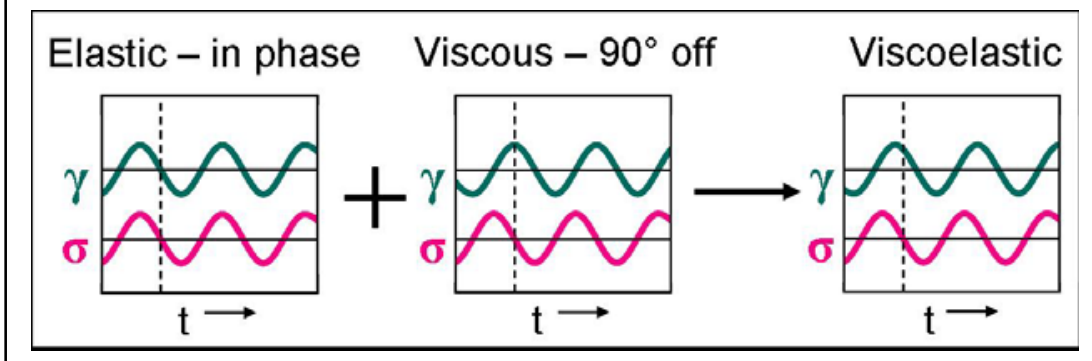

Figure 2.4. Oscillatory shear about a zero stress and zero deformation provides a method to characterize the elastic and viscous properties of tissue. The material properties given in the storage modulus ( $G^{\prime}$, elastic property) and loss modulus $\left(G^{\prime \prime}\right.$, viscous property) govern the relationship of stress $(\sigma)$, strain $\left(\gamma_{0}\right)$, and frequency $(\omega) . \quad \sigma=\gamma_{o}\left[G^{\prime} \sin (\omega t)+G^{\prime \prime} \cos (\omega t)\right]$

\subsection{Strengths and Limitations of Alternative Techniques: Illustration in Cornea and}

\section{Sclera}

An ideal goal of mechanical measurements would be to monitor the properties of tissue while under physiological conditions and stresses. The cornea and sclera are under stress due to the intraocular pressure of the eye, which results in tension of the tissue. During blinking the cornea experiences shear forces, and during eye movement, the sclera experiences shear, compression, and tension. When looking at the mechanical behavior of cornea and sclera, the majority of literature focuses on uniaxial tensile measurements. While this method has its use, other methods such as shear rheology and biaxial expansion can be used to further understand these tissues. In this section, we discuss some of the limitations and advantages of each method and how it applies to the particular problem of studying cornea and sclera. 
Extensive literature on tensile tests of cornea and sclera provides the majority of knowledge regarding the mechanical properties of these tissues. ${ }^{1-23}$ However, there are surprising differences between research groups' reported stress-strain curves and modulus values (e.g., Figure 2.5). Modulus values for human cornea were reported from $3.81 \pm 0.40 \mathrm{MPa}$ by $\mathrm{Zeng}^{2}$, up to $57 \pm 4.1 \mathrm{MPa}$ by Andreassen. ${ }^{3}$ Friberg has reported values for human sclera

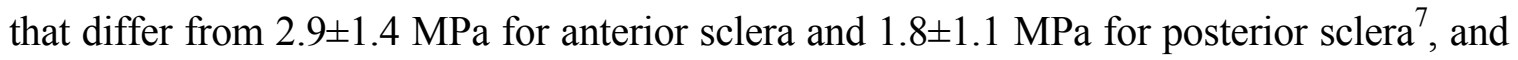
Wollensak reported values as high as $22 \mathrm{MPa}^{22}$ The differences in modulus values are due to differences in the particular measurement techniques and protocols used. While tensile measurements appear straightforward — cut a rectangular strip of tissue and apply tensionthe variations in the sample preparation, clamping, pulling, stress history, and hydration all play a role in the experimental outcome. 


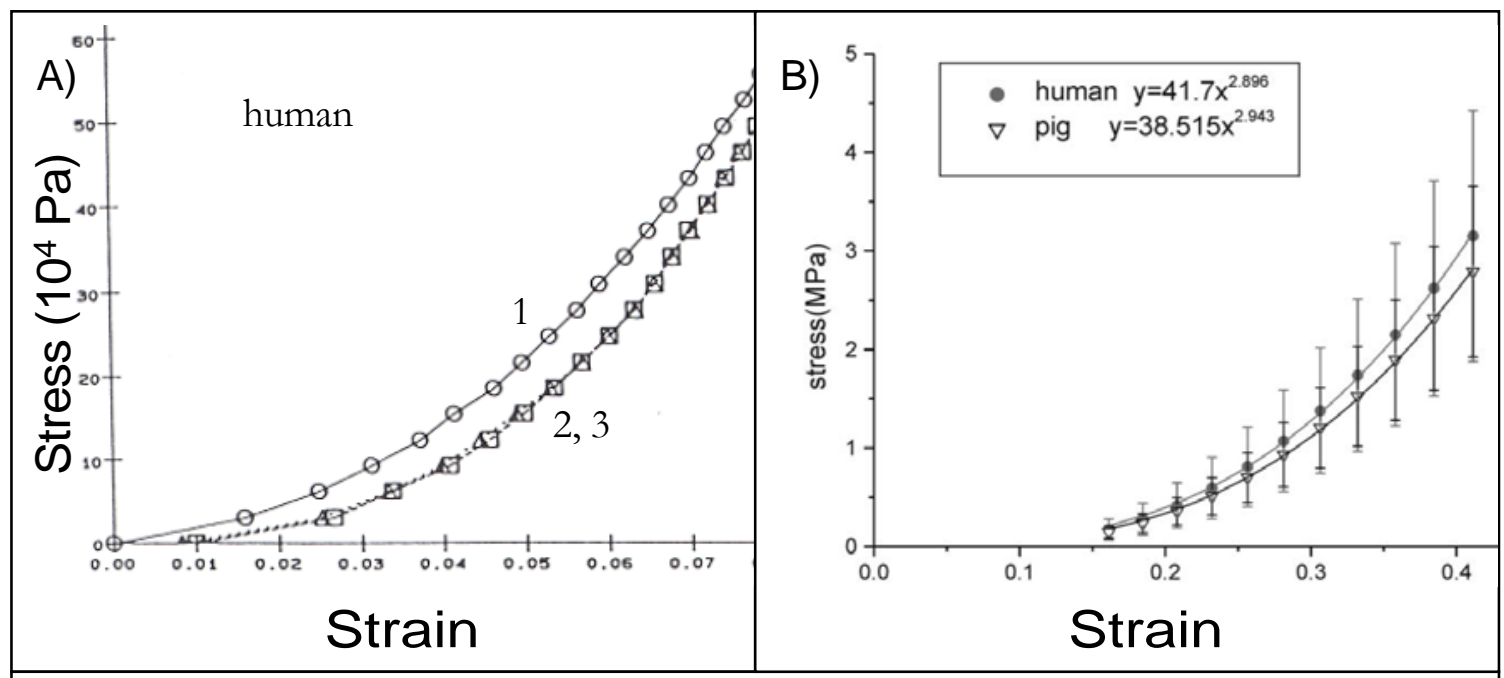

Figure 2.5. Results from (A) Hoeltzel ${ }^{1}$ and (B) Zeng $^{2}$ showing the stress-strain response of human cornea after cyclic conditioning. Hoeltzel demonstrates that 3 cycles removes hysteresis from the testing and Zeng shows results after 3 cycles. Note that results from Hoeltzel range from $0-7 \%$ strain and $0-0.5 \mathrm{MPa}$ stress while Zeng ranges from $15-40 \%$ stress and up to $3 \mathrm{MPa}$. This is exemplary of the vast differences that exist in current literature regarding mechanical testing of cornea and sclera.

Much of the variability comes from the difficulties associated with loading samples. The tissue hydration is important to consider, as tissue stiffness decreases as hydration increases, and swollen tissue may be more prone to failure. Different methods employ the use of dry or moist atmospheres, saline baths, and oil baths as ways of controlling the hydration.

In order to apply tension to the samples, the ends must be secured. Methods of securing the samples include clamping, gluing or pinning. Challenges associated with clamping result from slip of the sample in the grips and tissue damage near the ends due to deformation inherent in clamping. Challenges associated with glues include modification of tissue properties produced by glue that diffuses into the tissue and hardens. Lastly, pins cause further damage to the already cut samples. With all three fastening methods, further 
uncertainty in the measurement results from variability in configuration of the tissue between the fixtures. Ideally, the sample should be parallel to the direction of tension, and neither slack nor taut at the point of zero extension.

Unfortunately, samples are often slack upon loading, so there is uncertainty of the true length of the tissue. Several methods have been used to provide a self-consistent zero extension point for a set of measurements. For example, common practices include 1) alignment of data, 2) pre-stressing, and 3) minimal initial stress. Alignment of data uses features in the stress-strain curve (such as the elbow region) as registration points among a data set to shift individual curves on top of each other. After the curves are aligned, a zeroextension point is selected. Pre-stressing a sample involves imposing a specific, small stress for a specified time, after which the desired test condition is imposed (typically a specified crosshead speed or a specified load that is greater than the pre-stress load). The length at the end of the "pre-stress period" is taken to be zero extension and calculations of strain during the experiment relative to that length. The "minimal initial stress" method uses the extension at which the stress reaches a small, specific value during tension as the zero-extension point. In the experiments below, the minimal initial stress method was used.

The variability in loading methods, tissue preparation, and testing procedures all add to the uncertainty of results. The following experiments examine the variability of data that is seen under conditions that minimize the variability, namely low stress $(<1 \mathrm{MPa})$ and low strain $(<20 \%)$. To place the observed uncertainty in context, we compare two groups of specimens: sclera specimens held for 12 hours in saline (making them somewhat softer 
than their initial state) and specimens held for 12 hours in glyceraldehyde solution (making them much stiffer than their initial state). The experiments demonstrate the inability of this method to characterize the changes that result from fairly extreme crosslinking with glyceraldehyde.

Method: Eyes from 3-4 month old swine were ordered from Sierra for Medical Science and stored in saline on ice until use within 72 hours post mortem. Orbital tissues were removed and strips of either 3 or $5 \mathrm{~mm}$ width and approximately $20 \mathrm{~mm}$ length were cut from the cornea or the posterior sclera using parallel scalpel blades. The strips of cornea ran from superior to inferior across the center of the cornea (Figure 2.6a). The cornea was $\sim 14 \mathrm{~mm}$ in length, and surrounded on each side by $\sim 3 \mathrm{~mm}$ of sclera. The strips of sclera ran superior to inferior and were temporal to the optic nerve (Figure 2.6b). The control samples were stored in Dulbecco's phosphate-buffered saline (DPBS), while the treated samples were stored in a 2\% glyceraldehyde solution (GA). After 12 hours in solution at room temperature, the samples were tested on an Instron 5542 Universal Materials Testing Machine (Instron Corp., MA). The tissue sections were laid across the $6 \mathrm{~mm}$ gap of the testing apparatus as shown in Figure 2.7. To prevent slippage of the sample, pieces of Kimwipe were placed between the sample and the metal clamp, providing a higher friction surface. A permanent marker was used to mark where the tissue touched the clamp, and was observed for any movement that would indicate slip. After clamping, the samples were loaded vertically into the Instron and pulled at a rate of $1.0 \mathrm{~mm} / \mathrm{min}$ (cornea) or 1.5 $\mathrm{mm} / \mathrm{min}$ (sclera) while recording the stress. The samples were in air at $\sim 48 \%$ humidity and $\sim 20^{\circ} \mathrm{C}$. The instrument stopped pulling when a maximum load of $5 \mathrm{~N}$ was reached. Zero 
extension was taken as the point where the instrument first recorded a non-zero apparent stress $(0.005 \mathrm{MPa}=\mathrm{F} / \mathrm{A}$, where $\mathrm{F}$ is the measured load and $\mathrm{A}$ is the initial crosssectional area) in the sample.

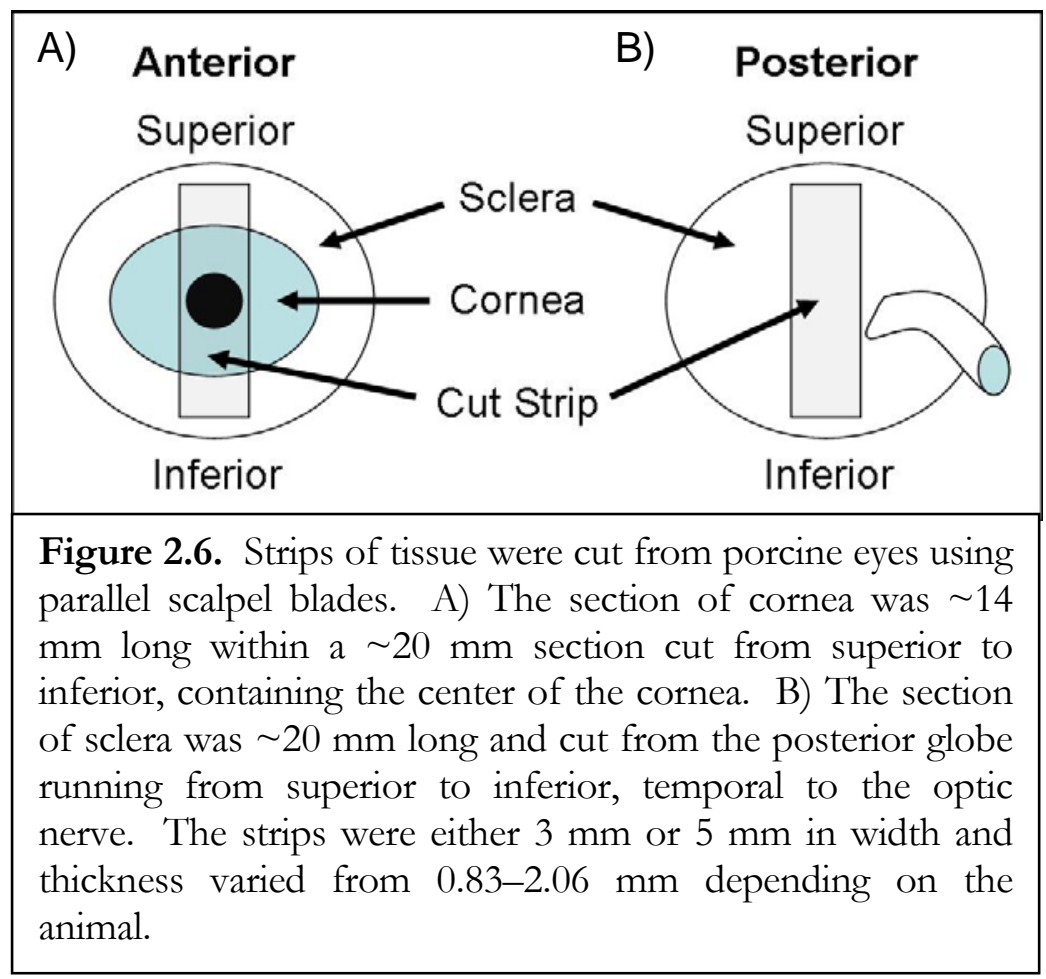




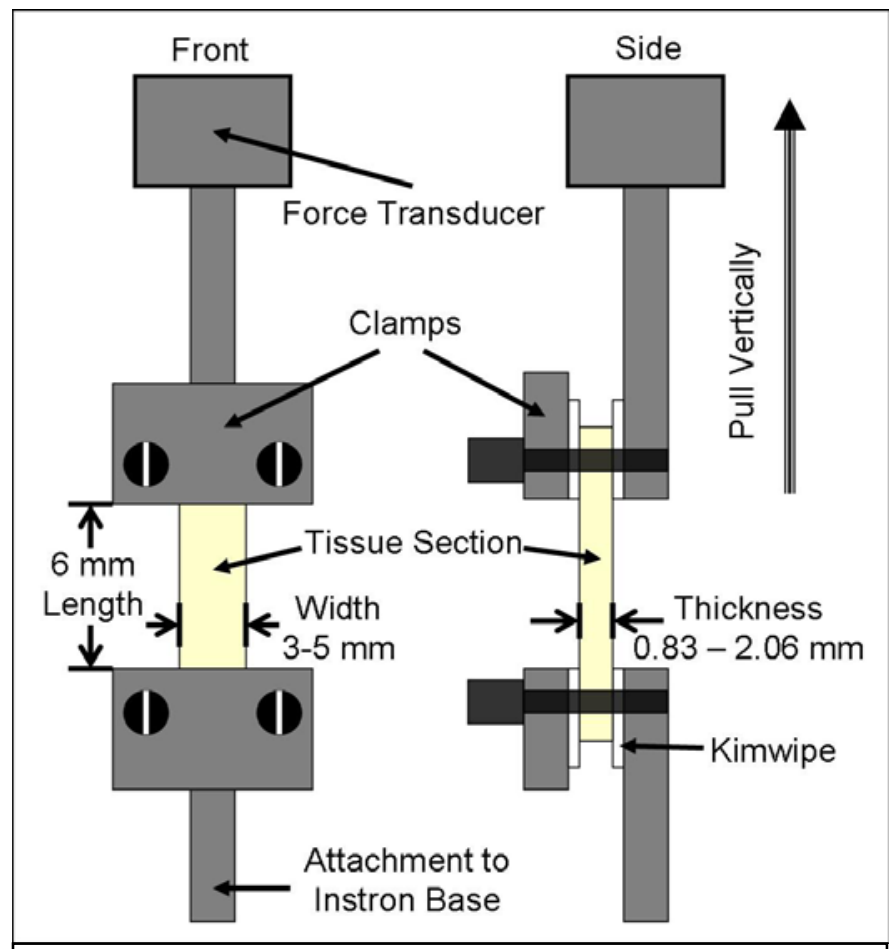

Figure 2.7. Tissue sections were mounted for testing on an Instron 5542. The specimens were laid across a $6 \mathrm{~mm}$ gap and clamped securely. Kimwipe between the clamps and tissue provided enhanced friction to prevent slipping. The clamp fixture was mounted vertically on the Instron and pulled at $1.0 \mathrm{~mm} / \mathrm{sec}$ (cornea) or $1.5 \mathrm{~mm} / \mathrm{sec}$ (sclera) while the force transducer recorded the load.

Results: Stress-strain curves of the scleral strips show large variability between different sections, as depicted in the scattered curves of control data (Figure 2.8a, b). Curves from 3 $\mathrm{mm}$ sections exhibit more scattering than those of $5 \mathrm{~mm}$ sections, but still exhibit a similarly shaped curve. The experimental soak with GA is extreme and generates changes in the tissue that are observable by eye and touch (tissue sections have browned and hold a curved shape during loading). It is expected that tensile tests would measure such differences, however, plotting the average results shows that there is no difference between 
the treated and control groups (Figure 2.9). In fact, the only significant difference is exhibited in low strain (0-5\%) where the $2 \%$ GA sections have less stress at the same strain as controls (significance determined from t-tests with $\mathrm{p}$ values $\mathrm{p}<0.05$ ). These results are contrary to observation and contrary to the known effects of GA. Because of the large variability that results from cutting, loading, clamping, and testing of samples, this method is not able to measure the change in modulus that results from GA treatment. This GA treatment would be more extreme than treatments planned for use in keratoconus or degenerative myopia. Because of the inability of this method to monitor large changes in treated tissue, other methods of characterizing tissue are discussed in the sections below. 


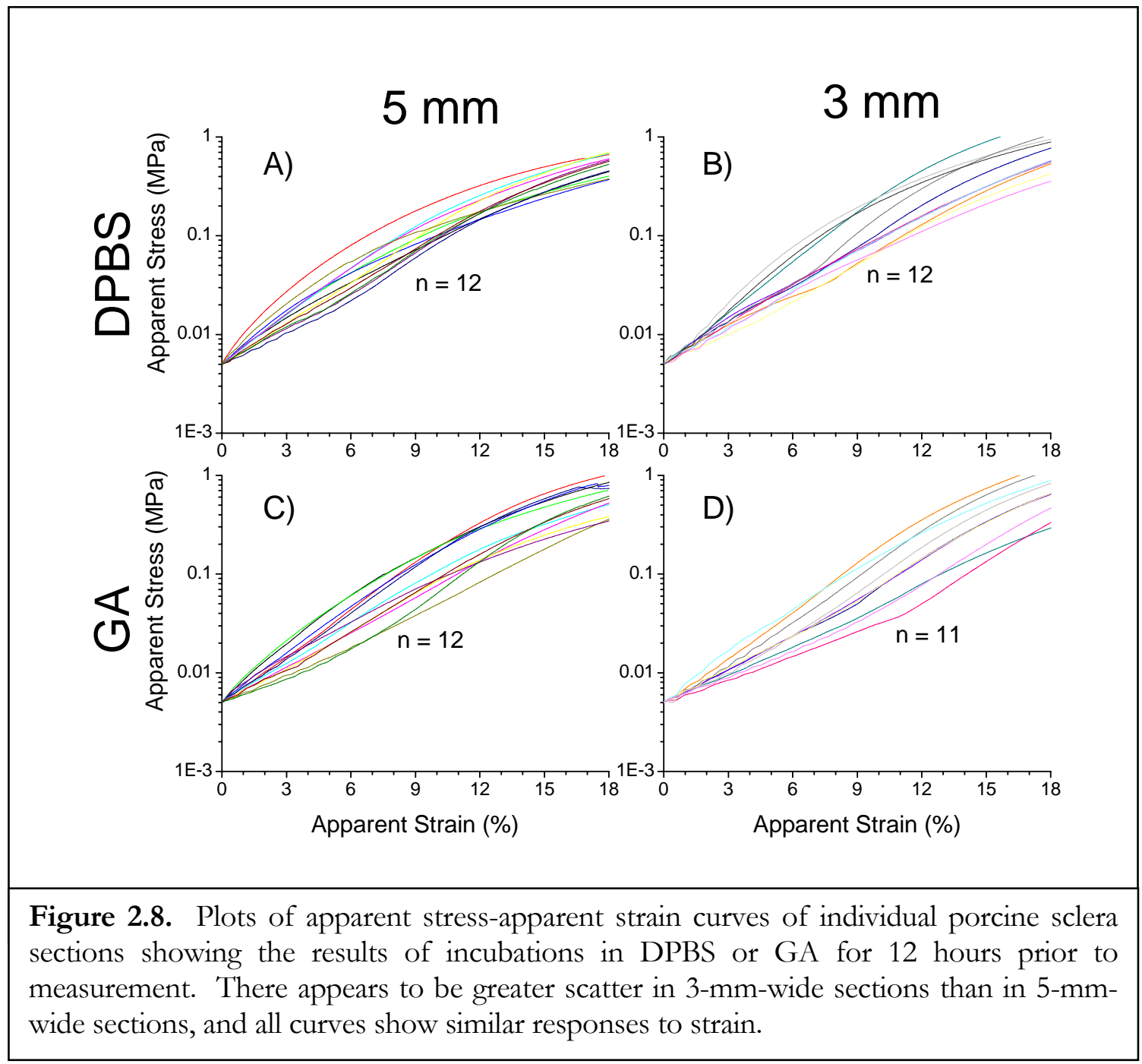



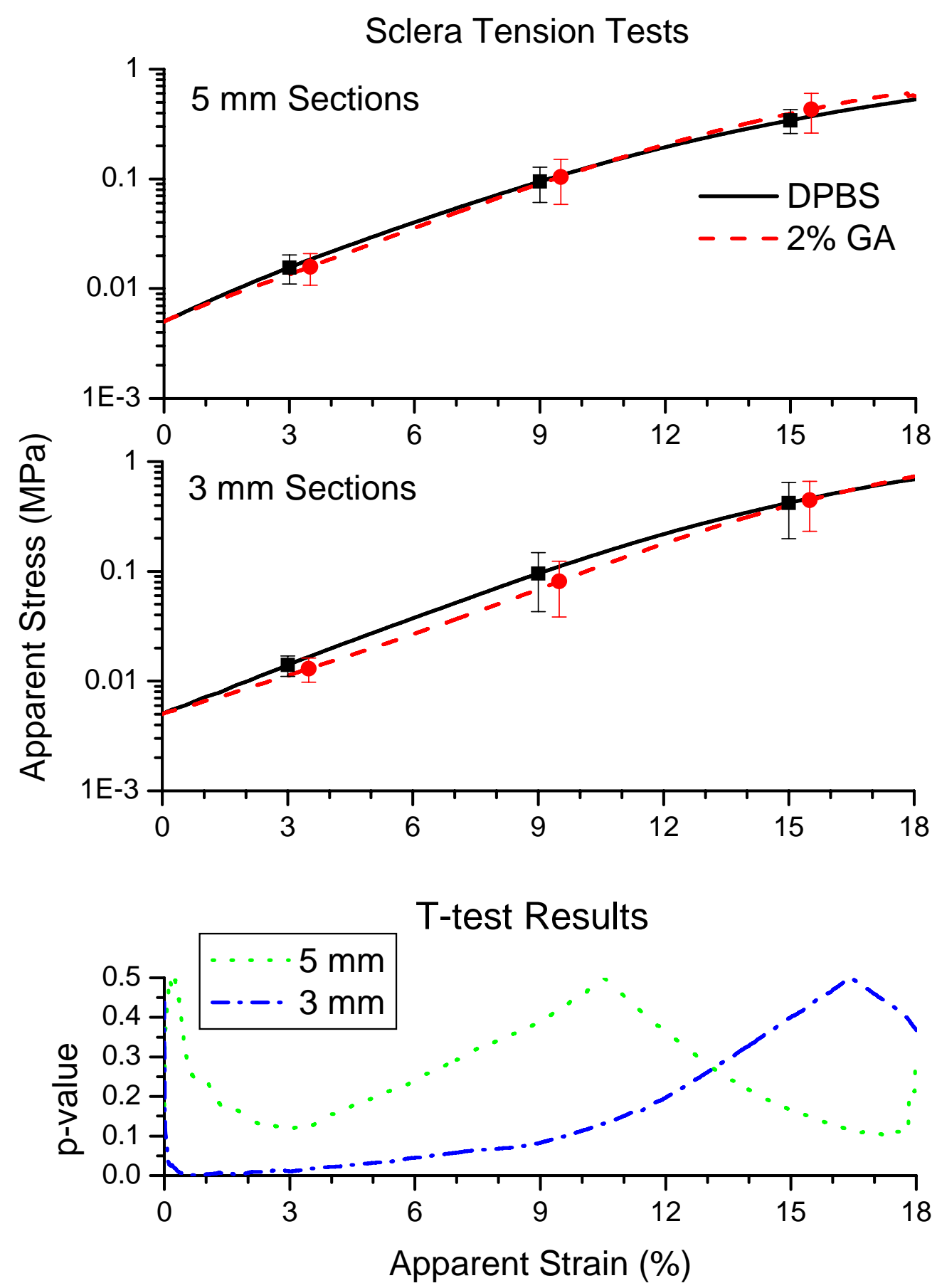

Figure 2.9. Averages of results displayed in Figure 2.8 indicate that there is no measureable difference in GA crosslinked sclera and control sclera. Although t-test results indicate that the GA treatment results in less stiff sclera for strains $0-5 \%$, this does not remain so for higher strains and is contrary to physical observation. These results are unreliable even when comparing groups of size $\mathrm{n}=11$ and 12 . 
The measured stress-strain curves for cornea are typical of those reported in literature and the graphs in Figure 2.10a demonstrate similar curves as in Figure 2.5. Out of 8 cornea strips tested, 5 did not have problems from loading, and individual curves are shown in Figure 2.10b. Cornea 2 shows little increase in stress above $10 \%$ strain, indicating either slippage, or failure of the tissue. Cornea 6 shows a bump at $17 \%$ strain which could also be explained by tissue slippage or failure. Although no signs of slippage could be seen from movement of the markings on the cornea, the individual curves indicate possible slippage. There is no way to determine if this is truly slip, so the data cannot be disregarded. With this in mind, the average and standard deviation that is displayed in Figure 2.10a could be unreliable and gives us uncertainty in the reliability of results published in literature. The same results are presented in Figure 2.11 with stress on a log scale. This scaling method allows examination of behavior at small strains, comparison to the porcine data, and it reveals that the scatter is increasing with strain rather than remaining a constant percentage of the stress. This scatter observed here is similar to the scatter reported for cornea in the introduction of this section.

Further examination of the variability due to different loading methods (specifically different prestresses) is easy to do using our extension curves. By changing the "minimal stress" used as a threshold for calculating zero extension, we change the observed curves (Figure 2.12). The curves become steeper and the tissue undergoes less strain before failure. The steepness of the curves is related to the elastic modulus, and from this example it is possible to see how having a higher prestress results in higher modulus values. The range of reported modulus values in literature can in part be attributed to the differences in 
prestress and loading techniques. It would be useful for future researchers to study the relative merit of different methods of securing samples and determining the initial sample length.
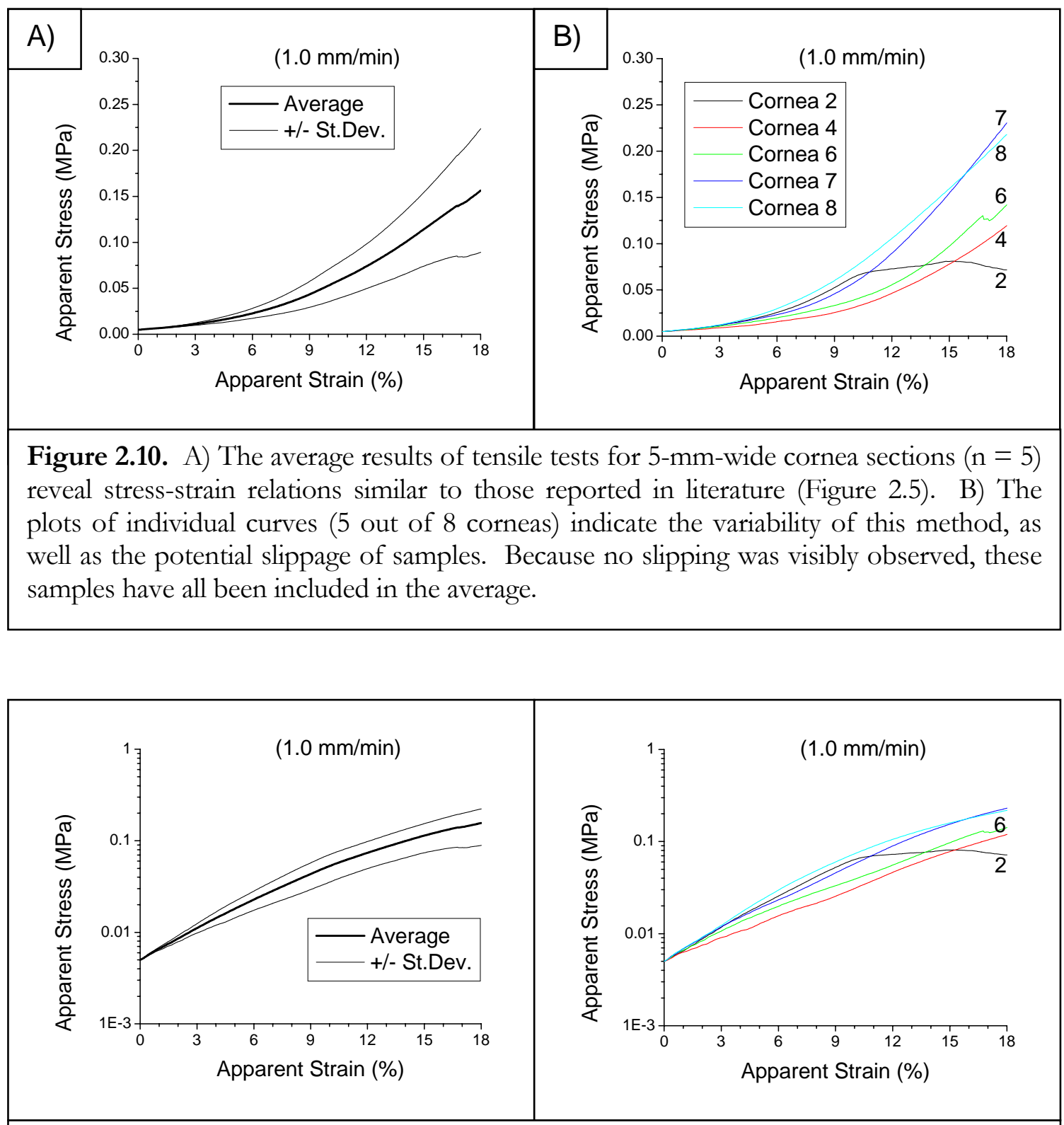

Figure 2.11. The same results presented in Figure 2.10 are shown here on a log scale. This scaling method helps demonstrate that scatter is increasing with increasing percent strain. 


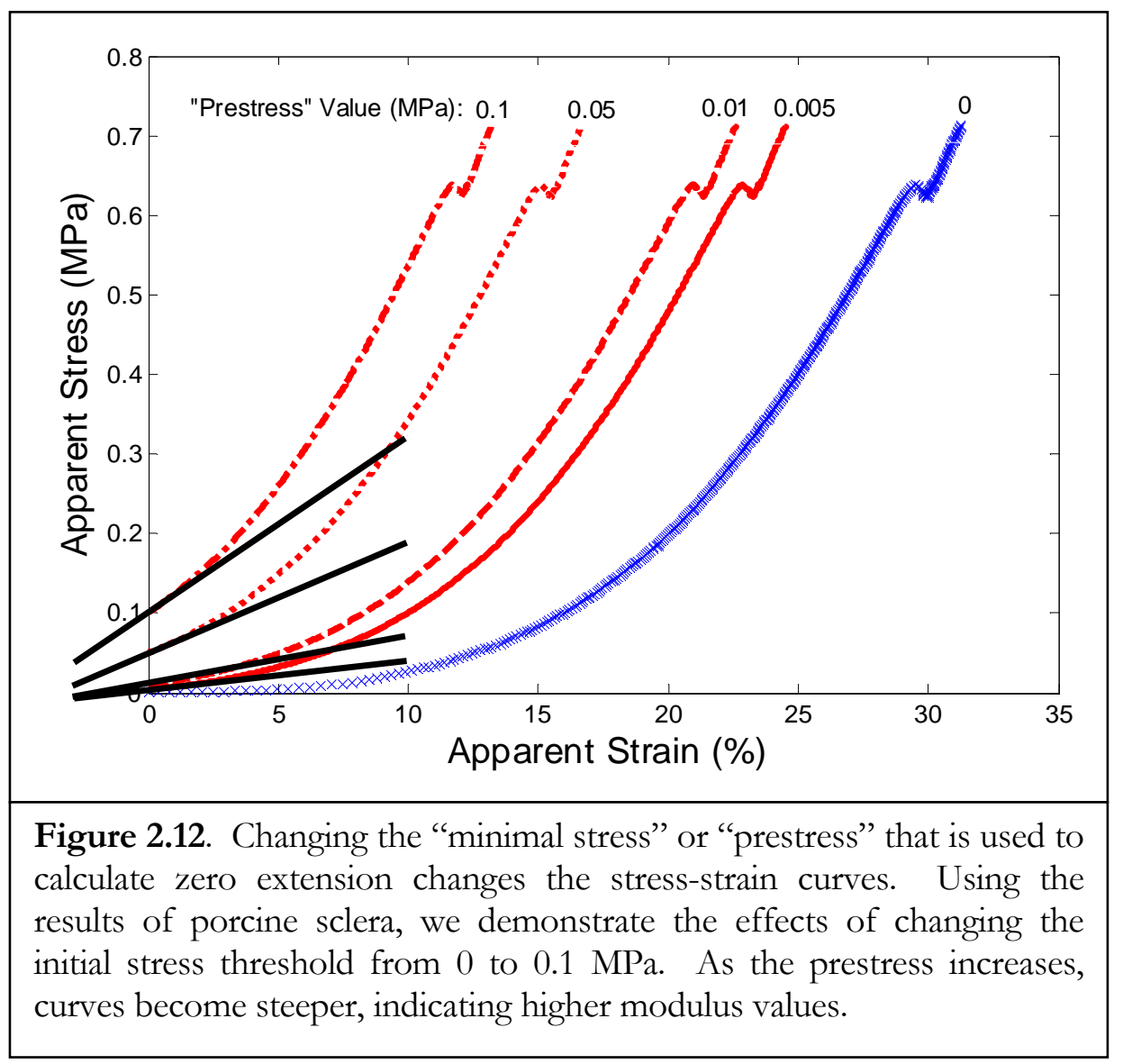

\subsubsection{Oscillatory Shear Rheology}

Shear provides complimentary information to elongation tests. Both the cornea and the sclera may be viewed as fiber-reinforced composites in which relatively tough, inextensible collagen fibrils are embedded in a soft hydrogel matrix. The fibrils are preferentially oriented in the plane of the tissue, with a greater degree of alignment in cornea than in the sclera. Under tension, the stress buildup occurs in fibers, while under shear the fibers are more prone to slide past one another. Therefore the apparent rigidity of the tissue is expected to be greater under tension than in shear. This hypothesis is referred to in 
literature about modeling the cornea and sclera $^{24}$, but there is limited literature which tests this hypothesis ${ }^{25}$.

Unfortunately, many of the sources of uncertainty encountered in tensile testing above are also confronted using shear; there are inherent difficulties in keeping tissue properly hydrated, avoiding structural damage while loading and testing, and imposing a welldefined deformation to a slippery specimen. Here we use a thermostated bath to control tissue hydration. We use a sample of radius $4 \mathrm{~mm}$ to analyze specimens cut from porcine eyes of radius $12 \mathrm{~mm}$, so that only small deformation is required to load the slightly domeshaped specimen into the disc-shaped geometry of the rheometer tools (Figure 2.13). To prevent slip, cleated tools developed in our laboratory ${ }^{26-29}$ are employed, providing superior results compared to smooth plates, roughened plates, or grooved plates. With these methods, reproducible measurements of the dynamic moduli were obtained. That is, oscillatory shear was imposed with such a small strain amplitude that the deformation did not perturb the structure or properties of the tissue. The present method allows for repeated measurement of storage modulus on the same scleral section (e.g., before and after crosslinking), and also enables in-situ monitoring of environmental effects on the modulus (e.g., effects of $\mathrm{NaCl}$ concentration, $\mathrm{pH}$, and temperature). 


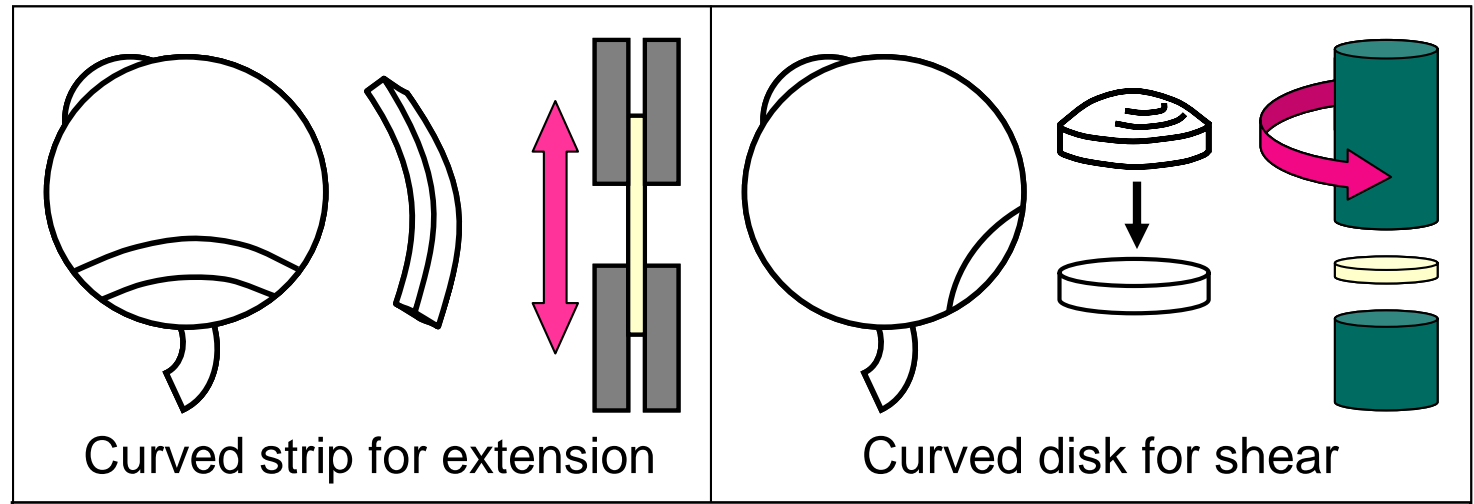

Figure 2.13. Strips cut for either tensile or shear measurements must be straightened before testing. Straightening the tissue introduces stresses in addition to those imposed by the measurement technique.

Materials: Dulbecco's phosphate-buffered saline (DPBS) was purchased from Sigma Aldrich $(\mathrm{pH} 7.5,0.9 \% \mathrm{NaCl})$. Solutions of varying salt concentration $(0-2 \%$ by weight $)$ were prepared by mixing $\mathrm{NaCl}$ in deionized water and adjusting the $\mathrm{pH}$ to 7.5 using $\mathrm{NaOH}$ or $\mathrm{HCl}$. A series of $0.9 \%$ saline solutions with $\mathrm{pH}$ ranging from 5 to 10 were prepared from $10 \mathrm{mM}$ PBS buffer- $4 \mathrm{~g} \mathrm{NaCl}, 0.72 \mathrm{~g} \mathrm{Na}_{2} \mathrm{HPO}_{4}$, and $0.12 \mathrm{~g} \mathrm{NaH}_{2} \mathrm{PO}_{4}$ (Sigma Aldrich) dissolved in distilled water for a final volume of $500 \mathrm{~mL}$ — with $\mathrm{pH}$ adjusted using $\mathrm{NaOH}$ or $\mathrm{HCl}$.

Porcine eyes were acquired from Sierra for Medical Science and were stored in $0.9 \%$ saline on ice until use within 72 hours of enucleation. The fat and muscle from all eyes were removed with scissors to expose the sclera. An equatorial incision was used to separate the posterior sclera from the anterior hemisphere of the eye. The vitreous, retina, and choroid were removed and $8 \mathrm{~mm}$ circular sections were cut using a trephine punch (Katena). The center of the circular section was located $\sim 10 \mathrm{~mm}$ from the optic nerve and in some cases 
multiple sections were cut from one eye. The following studies used approximately 60 eyes.

Procedures: Circular sections were tested in an $8 \mathrm{~mm}$ parallel plate geometry modified with cleats on an AR1000 (TA Instruments) Stress Controlled Rheometer (Figure 2.14). The sample was placed on the lower geometry, and then the upper geometry was lowered to contact the sclera. Experiments monitoring the effect of normal force and tissue compression on the modulus were used to determine the appropriate forces to achieve good contact (Figure 2.15). Good contact was made when the instrument registered a $0.1 \mathrm{~N}$ normal force. The tool position is then fixed and the gap between the tips of the cleats on the two tools was used to compute the shear strain from the observed torsional displacement. A small oscillatory stress was imposed on the sample such that the native structure of the tissue was not perturbed. The amplitude and phase of the resulting oscillatory deformation reveal the elastic and dissipative character of the material, which is typically a function of the frequency of the imposed oscillation. The elastic character of the tissue is manifested in the storage modulus, $\mathrm{G}^{\prime}(\omega)$. Because the sclera is solid, at low frequency its behavior is primarily elastic and the modulus becomes insensitive to frequency. Oscillatory shear measurements were done using a stress amplitude of $5 \mathrm{~Pa}$ and a frequency of $1 \mathrm{rad} / \mathrm{sec}$. Temperature was controlled using a Peltier plate and the solution surrounding the tissue was prevented from evaporating by using a solvent trap. The solvent trap had a port that could be accessed to change the solution around the tissue. 

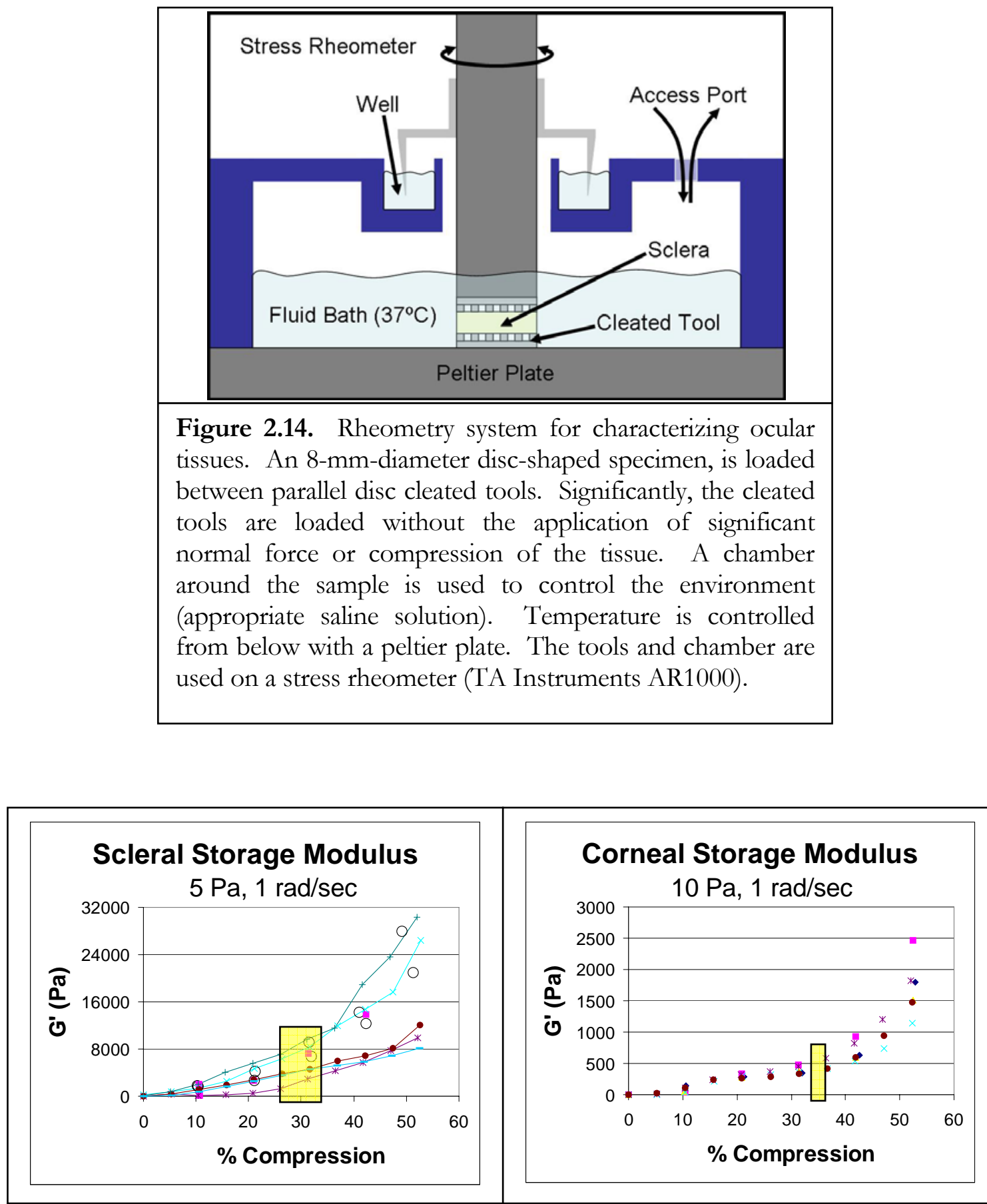

Figure 2.15. Modulus values increase as tissue is compressed. At low compression, the tissue is prone to slip, while at high compression residual stress builds in the tissue, artificially increasing the modulus. Loading with a normal force of $\sim 0.1 \mathrm{~N}$ compresses the sclera by $30 \pm 4.3 \%(n=12)$ and compresses the cornea by $35 \pm 1.6 \%(n=3)$. These regions of compression are indicated in the highlighted boxes on each of the graphs. Tissue was compressed to this point in order to ensure good contact and reliable modulus measurements without tissue slippage. 
Samples were tested using two different methods. First, the gentle nature of our loading protocol allows samples to be characterized pre- and post-incubation: with negligible damage, each sample can be loaded, measured for initial modulus, unloaded, incubated in a treatment solution, and measured on the rheometer again. Second, the effects of salt, $\mathrm{pH}$, and temperature on the modulus were measured in situ while leaving the sample loaded.

GA crosslinking: Using the incubation method, tissue sections were tested for changes in modulus due to GA crosslinking by measuring an initial modulus, soaking the tissue for 24 hours in $2 \%$ GA, measuring the modulus after treatment, rinsing for 24 hours in DPBS, and then measuring the modulus after treatment.

$\mathrm{NaCl}$ concentration: Testing for the effects of salt concentration was done using both an incubation protocol and an in situ transient protocol. For the incubation protocol, samples were soaked in DPBS for 24 hours, measured for initial modulus, incubated in $0-2 \% \mathrm{NaCl}$ ( $\mathrm{pH}$ 7.5) for 24 hours, and then measured for final modulus. An in-situ measurement of $\mathrm{NaCl}$ effects was conducted by continuously measuring the modulus while the bath fluid was changed (0-60 min $0.9 \% \mathrm{NaCl}, 60-120 \min 0 \% \mathrm{NaCl}, 120-180 \min 0.9 \% \mathrm{NaCl})$.

$\mathrm{pH}$ : The effects of $\mathrm{pH}$ on the scleral modulus were determined using both an incubation protocol and an in-situ protocol. The incubation method consisted of soaking samples in DPBS for 24 hours, measuring initial modulus, incubating in a $10 \mathrm{mM}$ PBS solution of a selected $\mathrm{pH}(5,6,7.4,8$, or 9$)$ for 24 hours, and then measuring a final modulus. In-situ measurement of $\mathrm{pH}$ dependence was done by continuously measuring the modulus while 
changing the surrounding solution (0-120 min DPBS at pH 7.5 and 120-140 min, 10 mM PBS of a selected $\mathrm{pH}[5,6,7,7.4,9$, or 10$])$.

Temperature: Temperature dependence was monitored in situ by continuously measuring the sclera at $37^{\circ} \mathrm{C}$ for 1 hour, lowering the temperature to $5^{\circ} \mathrm{C}$ for 1 hour, and raising the temperature back to $37^{\circ} \mathrm{C}$ for the final hour.

Results: The initial storage modulus of pig sclera as measured using a stress amplitude of 5 $\mathrm{Pa}$ and frequency of $1 \mathrm{rad} / \mathrm{sec}$ is $4500 \pm 1400 \mathrm{~Pa}(\mathrm{n}=66$ specimens cut from 22 eyes $)$ in DPBS at $37^{\circ} \mathrm{C}$. While our measurements of shear modulus were on the order of $5 \times 10^{3} \mathrm{~Pa}$, measurements of Young's modulus are of the order of $10^{6} \mathrm{~Pa}$ for sclera (previous section). In contrast to homogeneous isotropic materials, which have tensile modulus E only 3-fold greater than their shear modulus $\mathrm{G}$, the sclera show $\mathrm{E} \approx 100 \mathrm{G}$ consistent with the known structural anisotropy and inhomogeneity of the tissue noted earlier. While fibers are able to slide past one another in shear, the resistance to this motion arises from the components between collagen fibers and bundles (glycosaminoglycans, proteoglycans, and collagen connectors). Shear has the potential to provide information regarding the interaction of these components and how they contribute to strength.

GA crosslinking: Although the change in Young's modulus upon crosslinking with glyceraldehyde could not be successfully measured using tensile tests, shear measurements readily reveal an approximately 3 -fold increase in modulus after a 24 hour incubation period $\left(328 \pm 112.0 \%\right.$ increase in $\mathrm{G}^{\prime}$, Figure 2.16 , left). In contrast, the control specimens are slightly softer after their 24 hour incubation in DPBS. After a further 24 hour rinse in 
DPBS, the modulus continues increasing to $379 \pm 133.1 \%$ of its original value. The reason that GA crosslinking continues even after free glyceraldehyde is extracted and its implications regarding a clinical procedure are discussed in Chapter 3. The control specimens show a small further softening following their second 24 hour incubation in DPBS (Figure 2.16, right).

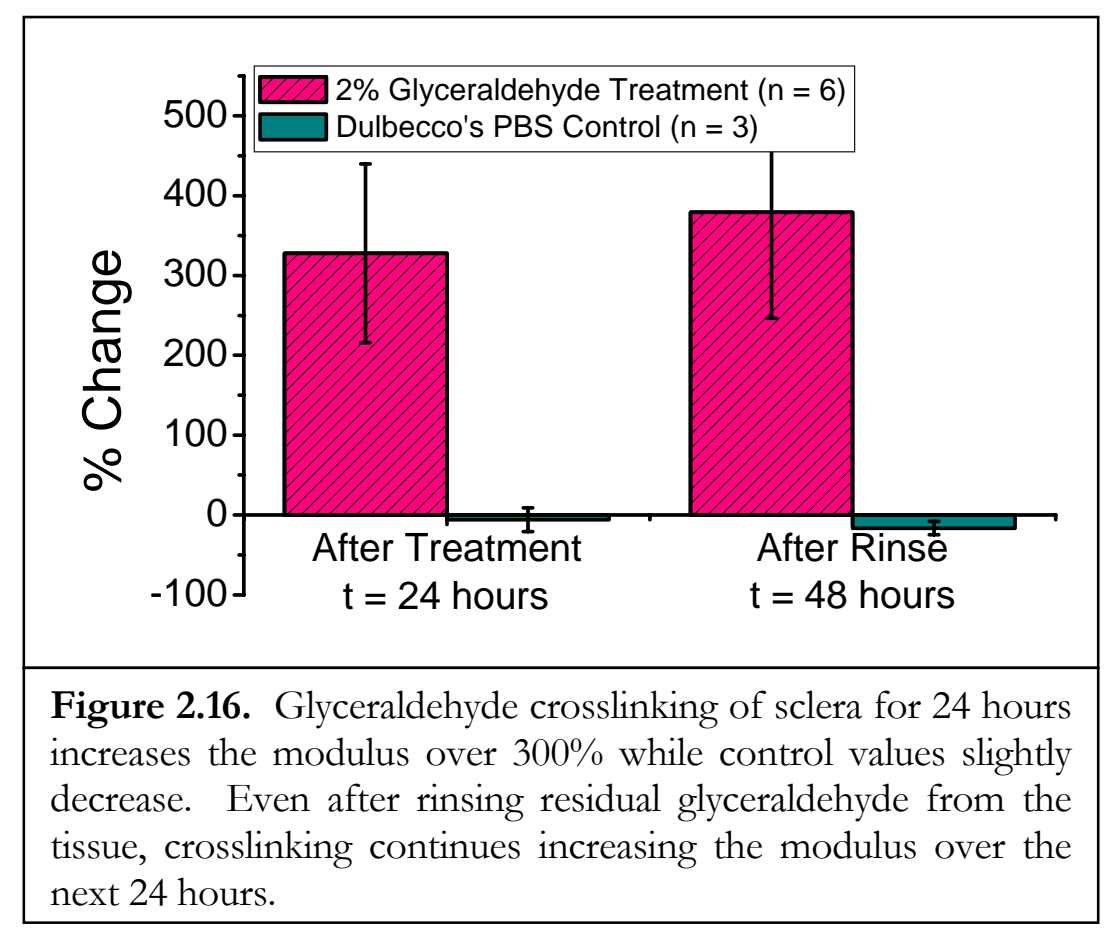

$\mathrm{NaCl}$ concentration: The effect of the salt concentration in the incubation solution on the change of modulus is pronounced at low salt concentrations (Figure 2.17). In particular, the shear modulus increases at least 4-fold when specimens are incubated in solution with $\leq 0.1 \%$ salt for 24 hours. Control eyes in DPBS show a slight decrease in storage modulus $(28 \pm 8.0 \%)$ upon the second measurement 24 hours after the first. Increasing the salt 
concentration causes an asymptotic decrease in modulus, reaching a $50 \pm 1.0 \%$ reduction in modulus at $2 \% \mathrm{NaCl}$.

In-situ measurements of shear modulus when the $\mathrm{NaCl}$ concentration is changed from $0.9 \%$ to $0 \%$ and back to $0.9 \%$ (Figure 2.18 ) demonstrate that change in modulus is reversible. In addition, the transient effect seen after changing the solution from $0.9 \%$ to $0 \%$ appears to plateau after $\sim 1$ hour. The transient effect upon replacing the $0.9 \% \mathrm{NaCl}$ solution occurs rapidly, with most of the effect occurring within 30 minutes. Permeability of the bovine sclera to sodium ions is large $\left(4.6 \times 10^{-5} \mathrm{~cm} / \mathrm{sec}\right),{ }^{30}$ and the transient response seen in the porcine sclera is likely due to diffusion of ions in and out of the tissue. The change in modulus is not accompanied by a change in normal force, which would indicate swelling of the bulk tissue.

The changes in modulus that occur with changes in salinity are most likely explained by the electrostatic screening effects of salt on the highly charged molecules within the sclera. Glycosaminoglycans are repeating units of disaccharides and are highly charged. Proteoglycans are protein cores with attached glycosaminoglycan chains. These molecules may be considered flexible while in high salinity, but as free electrolytes are removed, the glycosaminoglycan side chains expand, causing the proteoglycans to take on a stiff, "bottle brush" like conformation. Grodzinsky has proposed that the stiffening of molecules within the tissue (e.g., cartilage or sclera) would create stresses that are manifested as an increase in modulus. $^{31}$ 

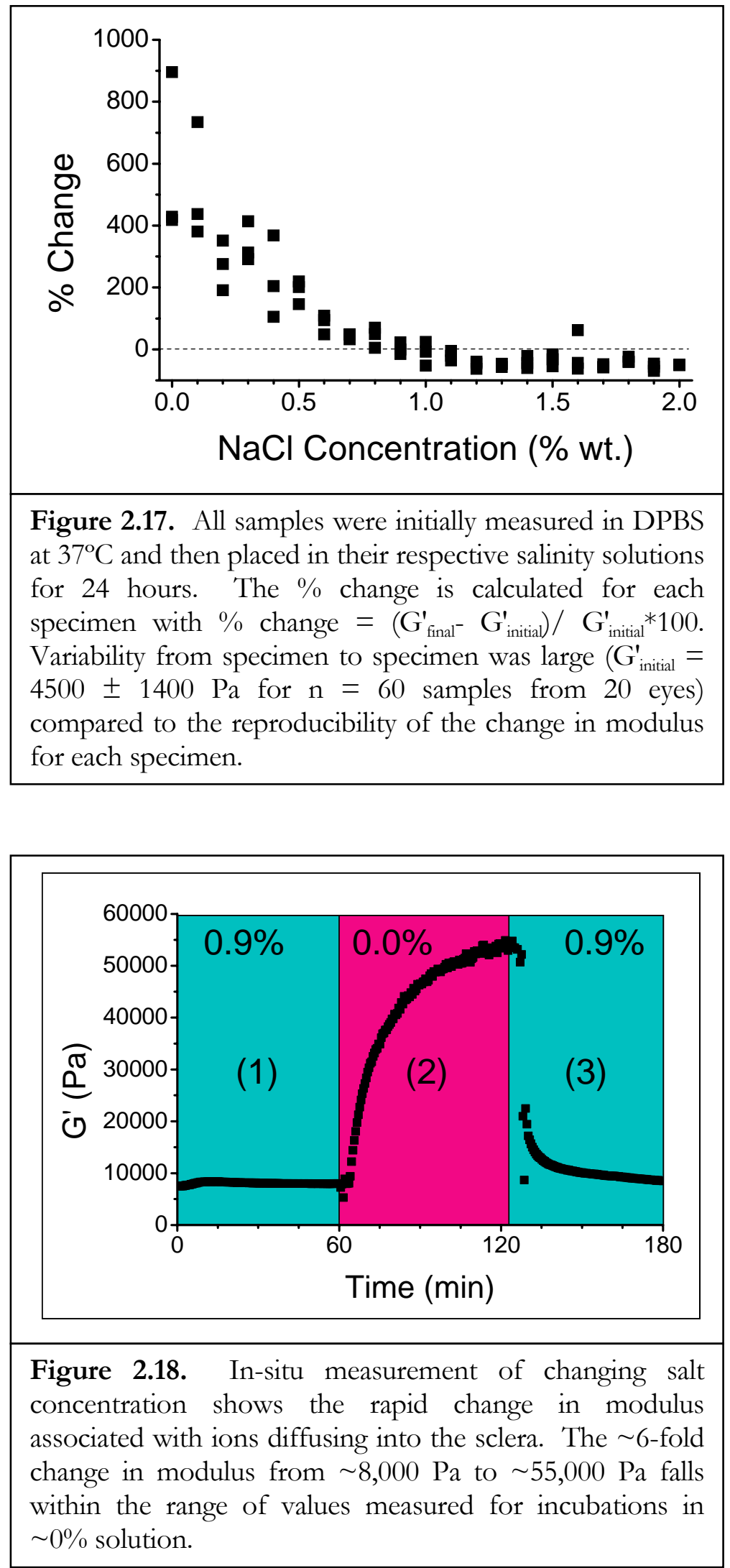
$\mathrm{pH}$ : Electrostatic interactions of the constituent molecules are also influenced by changes in $\mathrm{pH}$. Incubations of scleral discs in different $\mathrm{pH}$ solutions yield results given in Table 2.1. Increasing the $\mathrm{pH}$ does not cause any measurable differences as compared to controls at physiological $\mathrm{pH}$ of 7.4. However, decreasing the $\mathrm{pH}$ causes stiffening of the tissue, with a $48 \pm 40.1 \%$ increase in modulus at $\mathrm{pH} 5$. Note that the uncertainty present at $\mathrm{pH} 7.4$ contains variability of reloading, as well as sample-to-sample variability. The uncertainty of these measurements is large compared to the changes and makes conclusive observations difficult.

\begin{tabular}{|c|r|c|}
\hline \multirow{2}{*}{$\mathrm{pH}$} & \multicolumn{1}{c|}{$\begin{array}{c}\text { \% Change } \\
\text { Avg of }\end{array}$} \\
\cline { 2 - 2 } & $\begin{array}{c}\text { Avtdev } \\
\text { Samples }\end{array}$ \\
\hline 5 & $48 \pm 40.1$ & 9 \\
\hline 6 & $44 \pm 68.4$ & 6 \\
\hline 7.4 & $-21 \pm 23.6$ & 6 \\
\hline 8 & $-24 \pm 17.1$ & 9 \\
\hline 9 & $-24 \pm 19.3$ & 6 \\
\hline \multicolumn{2}{|c|}{ Table 2.1:24 Hour Incubation Experiments } \\
\hline
\end{tabular}

In-situ measurements remove the error associated with reloading a sample and definitively show the effects of altering the $\mathrm{pH}$. Lowering the $\mathrm{pH}$ increases the modulus while increasing the $\mathrm{pH}$ has little effect on the modulus (Figure 2.19). Within two hours, there is no change in modulus at $\mathrm{pH} 7.4,9$, or 10 . Although the modulus does not reach a plateau value within the 2 hours of this test, lowering the $\mathrm{pH}$ to 6 increases the modulus by $18 \pm$ $5.4 \%$ and lowering to 5 increases the modulus by $62 \pm 12.8 \%$. Based upon diffusion of ions in the $\mathrm{NaCl}$ studies, we would expect the transient response for $\mathrm{pH}$ changes to also near completion within 1 hour. Because the transient timescale is more than twice as long, we hypothesize that some form of remodeling occurs in the sclera. However, the modulus 
changes were reversible for all tested $\mathrm{pH}$ values, and Figure 2.20 demonstrates the reversibility with $\mathrm{pH} 7.4,5$, and 9 .

The pKa of sulfate, carboxyl, and amine groups that are relevant to proteoglycans and collagen in the cornea and sclera are $2-2.5^{32}, 4.1^{33}$, and $9.3^{33}$ respectively. As $\mathrm{pH}$ approaches these values, we expect to see changes in the mechanical properties. While we would expect large effects close to these values, it is interesting to notice that there is no response when passing the $\mathrm{pKa}$ for amines. At lower $\mathrm{pH}$, the sulfate and carboxyl groups are expected to become protonated, reducing charge in the tissue. A reduction in charges could reduce the stress of the molecules in the tissue and reduce the modulus, so the increase in modulus at $\mathrm{pH} 5$ and 6 is counterintuitive. Future research into the interactions within the sclera is necessary to explain these results.

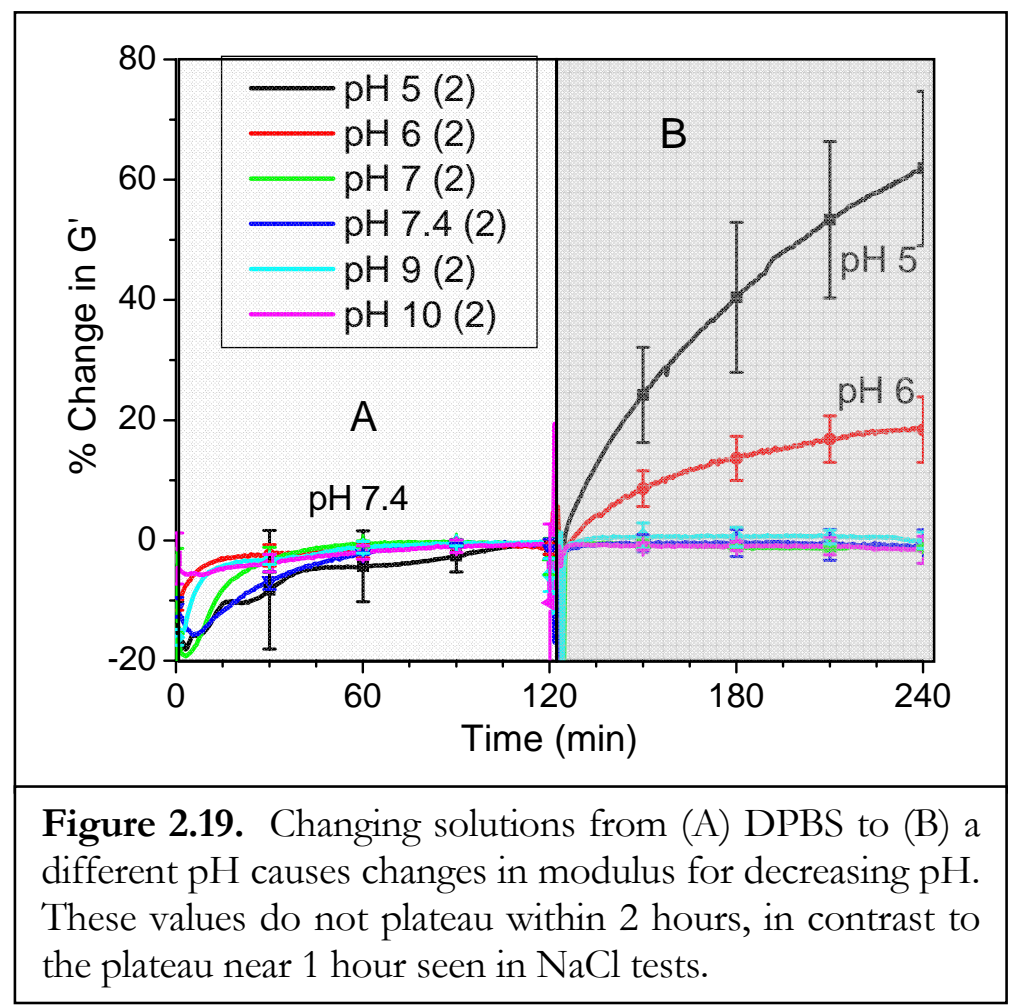




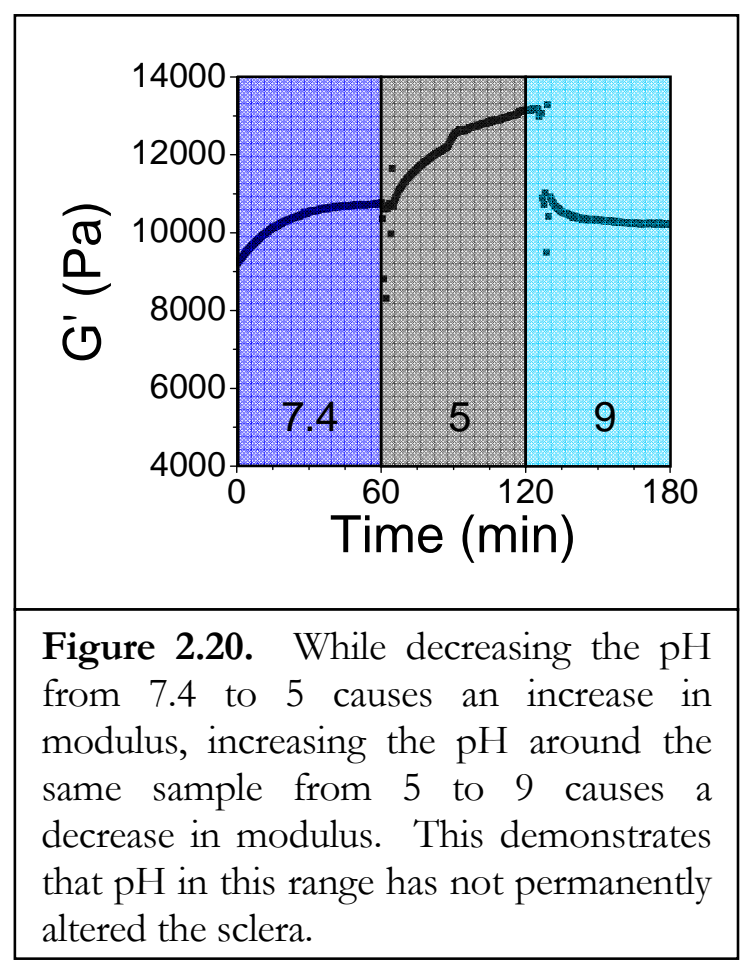

Temperature: Cooling of samples from $37^{\circ} \mathrm{C}$ down to $5^{\circ} \mathrm{C}$ causes more than a $60 \%$ increase in modulus (Figure 2.21). This finding is in agreement with prior literature regarding the softening of tissue with increasing temperature. ${ }^{25,34}$ 


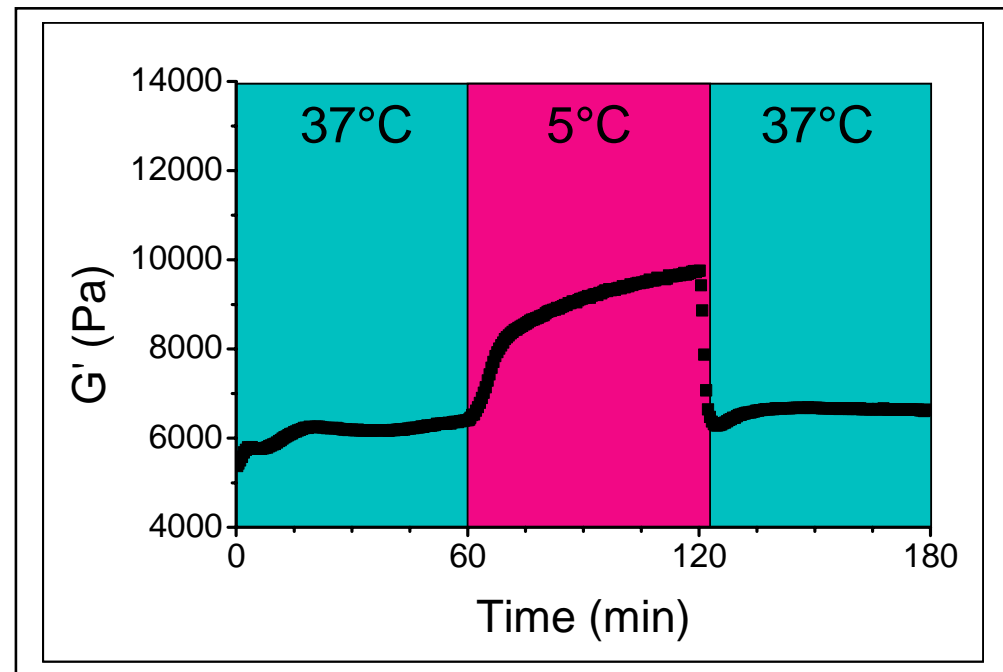

Figure 2.21. The modulus increases as temperature is decreased and causes stiffening of the tissue. Returning the temperature to physiological levels restores the modulus to its original value.

In-situ monitoring helps eliminate some of the variability associated with repeated loading and unloading of a sample, and provides a more accurate method to study the effects of environmental factors on the tissue. Shear measurements are subject to similar variability of cutting and loading specimens as tensile tests. This is clear from the large scatter in initial modulus measurements $(\mathrm{G}=4500 \pm 1400 \mathrm{~Pa})$. The ability to non-destructively measure and remeasure samples provides a way to remove some sample-to-sample variability. Samples used for measurements of $\mathrm{NaCl}$ concentration effects show remarkable similarities in $\%$ change. Changing from repeated measurements to in-situ monitoring further reduces the variability by eliminating errors introduced by reloading the sample. In-situ monitoring very clearly shows the time course of changes that happen in individual specimens. 
The uniaxial tensile tests and shear tests mentioned above do not accurately simulate the loading geometry that occurs in vivo. The sclera and cornea are under tension due to the IOP, which imposes an approximately biaxial stress, not a uniaxial one. Shear stress can be imposed during blinking and eye movement, but it is in addition to the biaxial tension that is constantly acting on the cornea and sclera. To more closely mimic the physiologic stresses, researchers have used button and intact globe expansion tests. ${ }^{34-49}$

Button tests consist of removing a portion of the eye and clamping it to a pressurizable chamber (Figure 2.22a). The clamps around the specimen periphery are to ensure a fixed boundary condition as the interior surface expands under pressure. Because of the cutting and mounting of specimens, this method has disadvantages similar to the tensile and shear tests. 


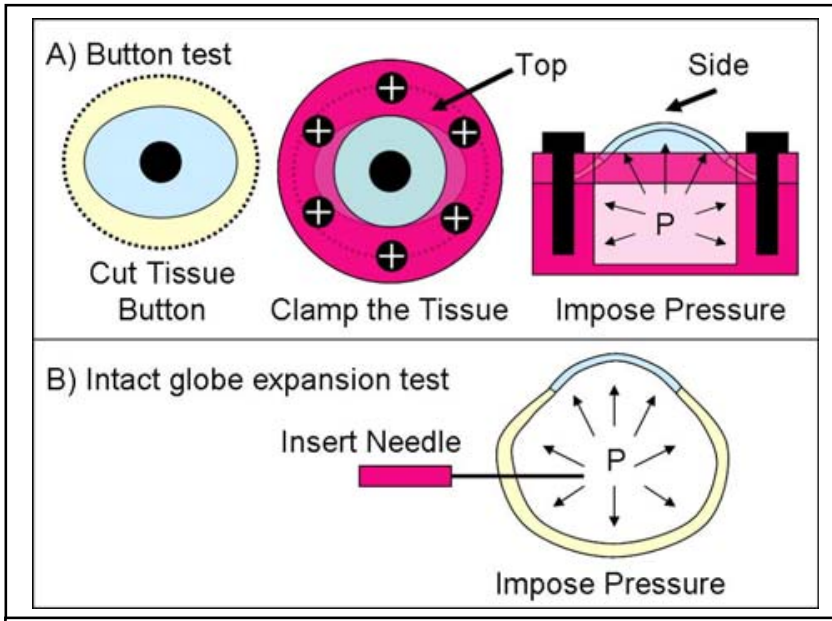

Figure 2.22. A) Buttons of tissue cut from the eye are mounted on a fixture with a circular hole that allows tissue to expand outward. The tissue is clamped down and the pressure inside the fixture is elevated to cause expansion. B) The entire eye is kept intact and a needle is inserted to control intraocular pressure.

Intact globe expansion experiments use the eye without cutting the tissue (Figure 2.22b). The fibril structure is not disturbed by cutting or clamping, and there is an attempt to maintain the natural boundary conditions of the eye. Most importantly, the type of stresses imposed in this intact globe expansion test provide a model for conditions that more closely resemble in vivo stresses. Some research groups have glued eyes to fixtures, or pierced eyes with several needles in order to hold them steady while testing. In our experiments, whole eyes are set upon a ring support and pierced through the posterior pole with a single needle that is used to control IOP. In this manner, there is only minimal damage at the site of the needle. The eyes are not clamped or glued to the ring support, so they are free to expand along any ocular dimension. To measure ocular dimensions, groups have used lasers, interferometry, ultrasound, calipers, and photography. With current advances in 
technology, cheap cameras are able to provide high-resolution images $(\sim 28$ micron/pixel in this study) and even video. Using this method, we are able to keep the eye submersed in a physiological bath and remotely monitor the shape change. By minimizing tissue disturbance, variability is reduced to that associated with animal-to-animal variability.

In degenerative myopia and keratoconus, there are shape changes that occur gradually under a normal intraocular pressure. While creep occurs in the weakened, diseased tissue in vivo, we are able to use intact globe tests to induce creep of cornea and sclera by imposing elevated intraocular pressures in distensible rabbit eyes in vitro. By inducing creep, we are able to study the deformation of the tissue, and are able to study treatments for their ability to prevent deformation. We demonstrate eye creep at low and high IOPs and demonstrate stabilization of eye shape using crosslinking with GA.

Materials: Eyes from New Zealand White rabbits were provided by Keith Duncan at University of California, San Francisco. Because young eyes are more distensible (collagen fibrils are not completely matured), we have used rabbit kit (2-3 weeks old) globes for these experiments. The eyes were stored in saline on ice until use within 48 hours of enucleation. The fat and muscle from all eyes were removed with scissors to expose the sclera. Twelve rabbits were used in this study.

Procedures: Enucleated eyes used for the study of elevated pressure and creep were placed in DPBS at room temperature $\left(\sim 22^{\circ} \mathrm{C}\right)$ for 30 minutes prior to loading. GA crosslinking was done by removing the corneal epithelium, soaking for 12 hours in $2 \%$ GA, rinsing for 
12 hours in DPBS, and then loading. Eyes were loaded in a transparent plexi-glass observation cell filled with DPBS. The eyes were aligned with the major axis of the equator parallel to the imaging plane. Two holes at the bottom of the cell were used for the insertion of hypodermic needles (30 gauge) that regulate the IOP (Figure 2.23b). The needles were inserted into the eyes through the posterior sclera, and they were fed from a DPBS reservoir held a certain height $h$ above the apparatus, thus providing an IOP governed by hydrostatic pressure (IOP $=\rho g h ; \rho=$ density, $\mathrm{g}=$ gravitational acceleration). The eyes were equilibrated in the cell for 15 minutes without any control of IOP. The pressure valve was then opened, and the eyes were maintained at an initial IOP of 22 $\mathrm{mmHg}$ for 1 hour, and then maintained at either 22 or $85 \mathrm{mmHg}$ for 24 hours. Photographs of the eyes were taken every 15 minutes throughout the experiment and then analyzed for changes in ocular dimensions using MATLAB (Scleral Perimeter-SP, Equatorial Diameter-ED, Scleral Length-SL, Corneal Perimeter-CP, Corneal Diameter-CD, Corneal Length-CL, and Axial Length-AL, Figure 2.23c). The Canon Powershot G3 used in these experiments gave a resolution of $\sim 28$ microns per pixel. For the 24 hours preceding the experiments, the DPBS was set out at room temperature so that dissolved gasses could escape and eliminate bubbles that interfere with measurements. To minimize bacterial growth during the experiment, several antibiotic eyedrops (Bausch \& Lomb neomycin, polymyxin B sulfate and gramicidin ophthalmic solution USP) were added to the solution in the observation cell. 


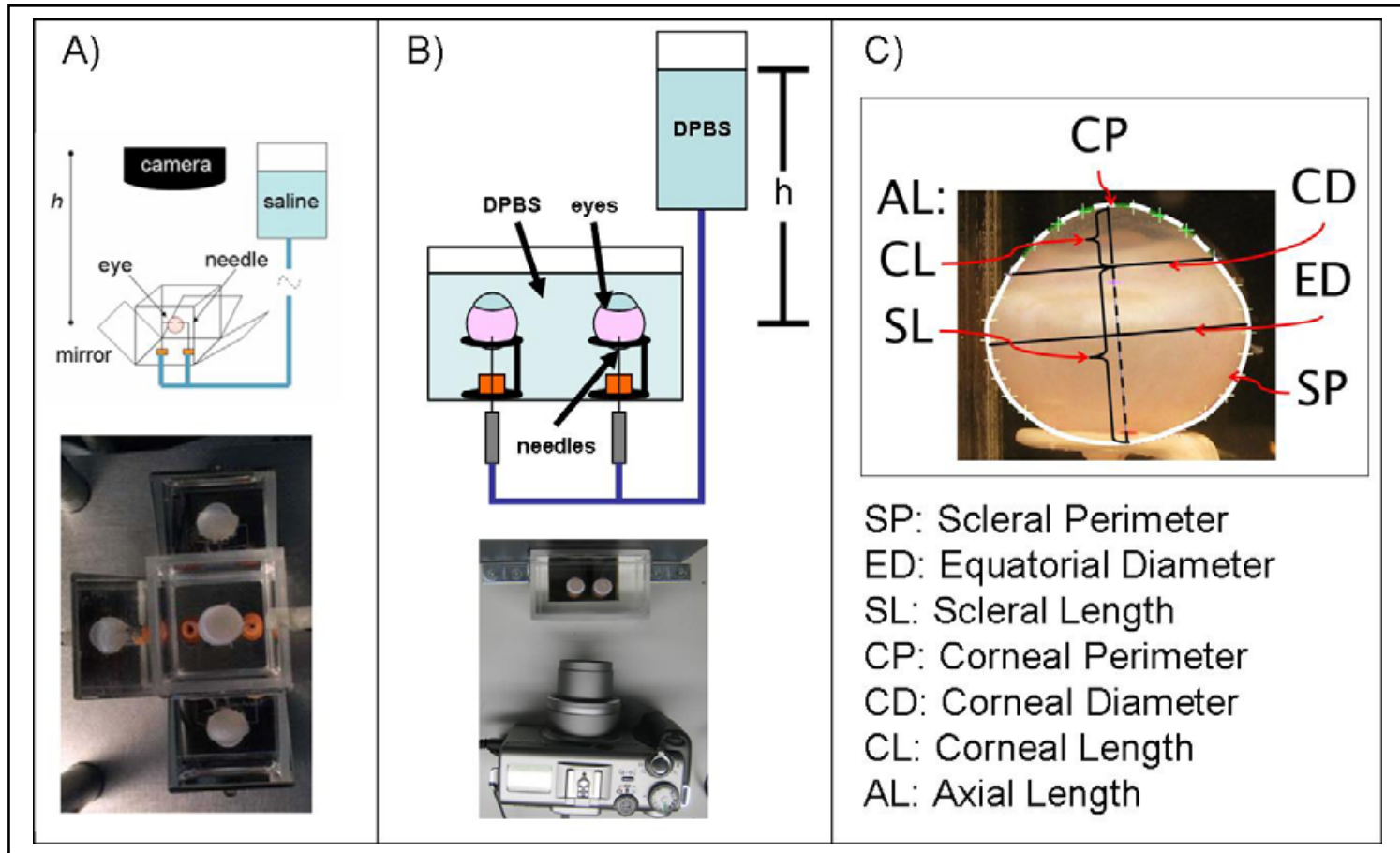

Figure 2.23. A) Initial setup using mirrors for photographing an eye from multiple angles to give a more complete record of eye expansion. This was traded for the simplicity of the setup in B) which uses a pair of eyes for internal control and a ring stand that allows free expansion of the eye. C) Ocular dimensions are measured using custom MATLAB software.

Results: Because the enucleated rabbit eyes are taken from saline and loaded without application of pressure, they are initially in a slack, unknown configuration. After turning on the low pressure $(22 \mathrm{mmHg})$, the eyes have a fast expansion that takes place within the first 30 minutes and is mostly complete by the time 1 hour has elapsed (Figure 2.24a). As an example, results for $\mathrm{CP}$ of 5 eyes show a jump in length from the second to third data points, coinciding with the application of pressure. It is interesting to note that eyes 1 and 2 (belonging to the same animal) have initial differences in shape that are reduced after pressure is applied. The difference within animals $(1 \& 2$, or $3 \& 4)$ is much smaller than the variability of the entire group, indicating that fellow eyes provide a good control for these studies. Looking at the percent change in shape from the onset of low pressure 
demonstrates that there is large scatter in the end results, with measurements ranging from $6-22 \%$ after 24 hours (Figure 2.24b). For studies of creep, which are applicable to keratoconus and degenerative myopia, we are interested in the changes that occur after the initial expansion period. The creep is determined from the changes occurring after 1 hour of low pressure application (Figure 2.24c). We see that creep of the eyes has small variability ( $0-6 \%$ change) compared to the expansion from the initial shape. All but one of the eyes fall in the range of $4-6 \%$ change. The method of applying a low pressure for a specified time is similar to applying a prestress in tensile tests. However, the boundary conditions of a whole eye ensure the prestress is distributed similarly to in vivo stresses. This prestressed configuration ( 1 hour at $22 \mathrm{mmHg}$ ) is taken as the starting point for further analysis of creep. Measurements of eye dimensions after 1 hour of low pressure give a baseline for the initial size of the rabbit eyes (Table 2.2). Equatorial diameter (ED) is larger than the axial length, indicating the oblate shape of the eyes. 


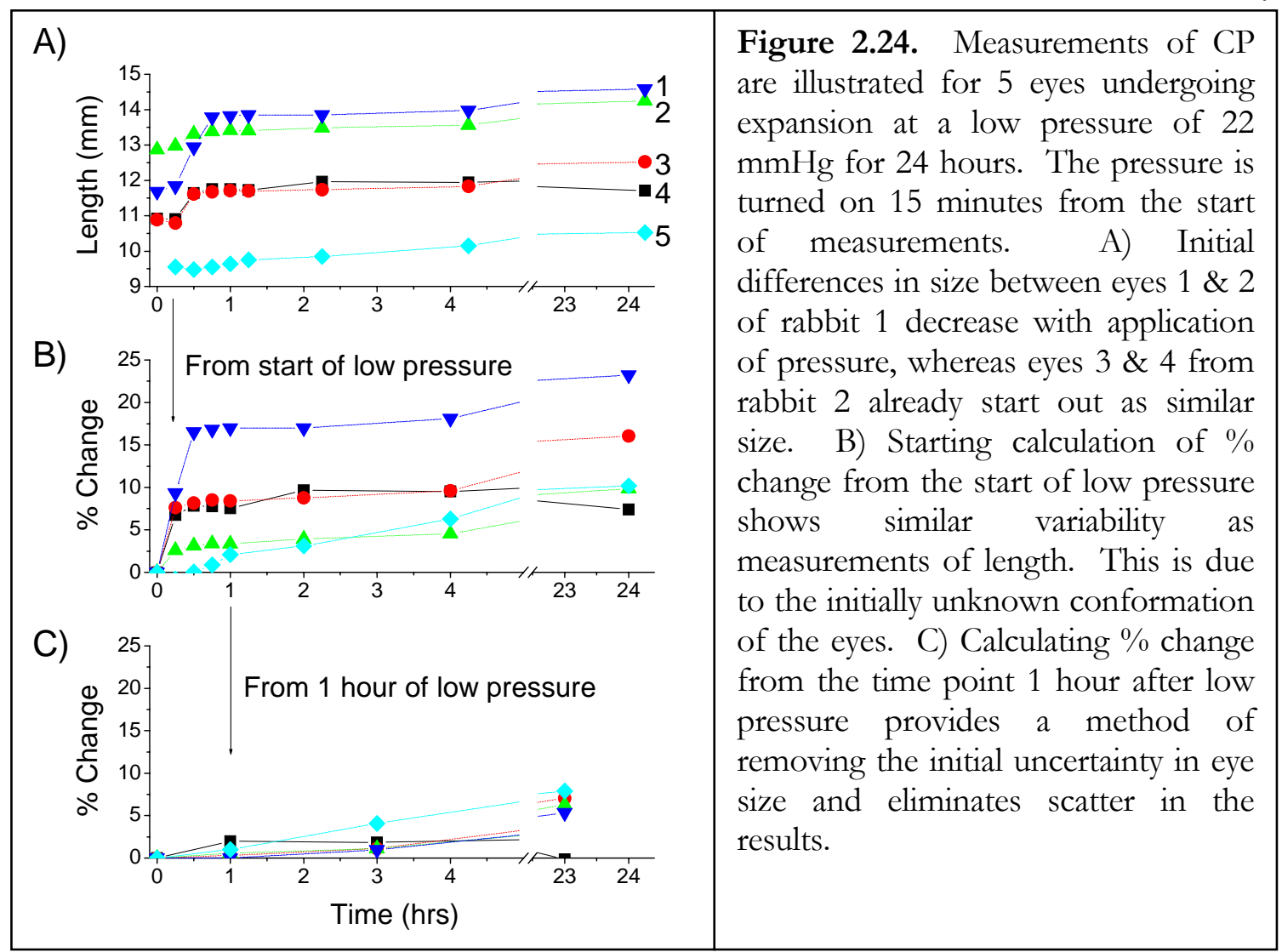

\begin{tabular}{|c|c|c|}
\hline \multicolumn{3}{|c|}{ Ocular Dimensions } \\
1 Hour at $22 \mathrm{mmHg}(\mathrm{n}=11$ eyes $)$
\end{tabular}


After applying the initial prestress, maintaining the eyes at low pressure for up to 24

hours induces less creep than maintaining the eyes at high pressure (Figure 2.25). The cornea and sclera behave differently, as can be expected from tensile tests which demonstrate the sclera has a higher modulus. At low pressure, it is apparent that the sclera is less susceptible to creep than the cornea (SP, ED, \& SL change $<1 \%$ while $\mathrm{CP}, \mathrm{CD}, \&$ CL change 4,2 , and $10 \%$ respectively). Together, the cornea and sclera contribute to the changes in $\mathrm{AL}(2 \%)$. The changes in $\mathrm{CP}, \mathrm{DP}, \mathrm{CL}$, and $\mathrm{AL}$ do not appear linear and may reach plateau values in a period longer than 24 hours. For the high pressure, the creep rates appear linear from 8-24 hours. 

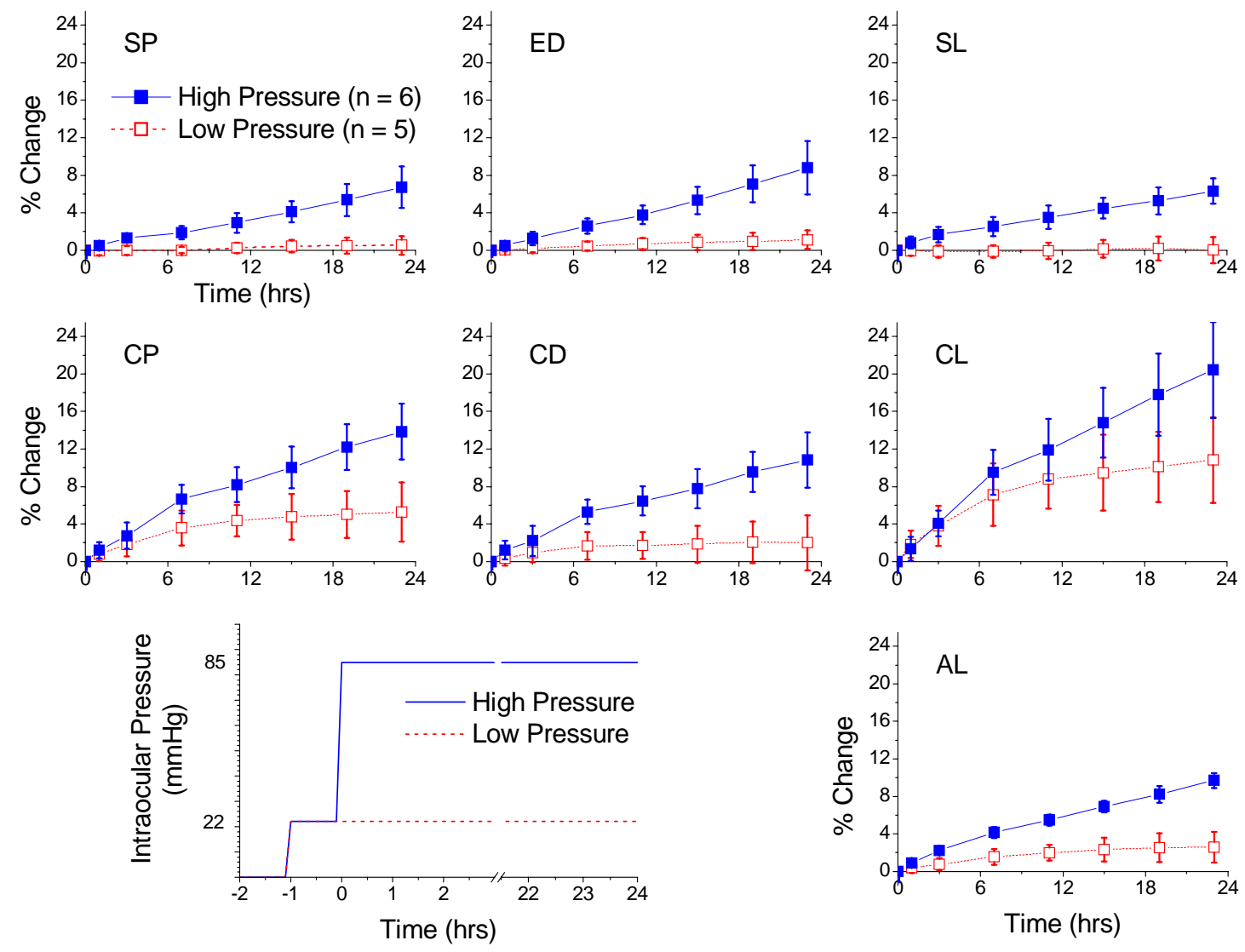

Figure 2.25. Rabbit eyes subjected to low $(22 \mathrm{mmHg})$ and high $(85 \mathrm{mmHg})$ pressures for up to 24 hours show the susceptibility of the cornea and sclera to creep. At low pressure, the sclera remains stable (top row) while the cornea expands to nearly a plateau value (middle row). At high pressure, the cornea and sclera both expand and the creep rate appears linear from 8-24 hours. For values of the overall dimensions, refer to Table 2.2.

The continuous creep experienced in the high-pressure eyes is useful for the study of keratoconus and degenerative myopia where we are trying to examine tissue that progressively changes shape. Further, treatments that successfully prevent expansion of eyes due to creep could have potential as therapeutic agents. Eyes soaked in 2\% GA treatment became hard and yellow, indications that crosslinking had occurred. After 24 hours at the high pressure, none of the treated eyes expanded, while control eyes expanded in all dimensions (Figure 2.26). 

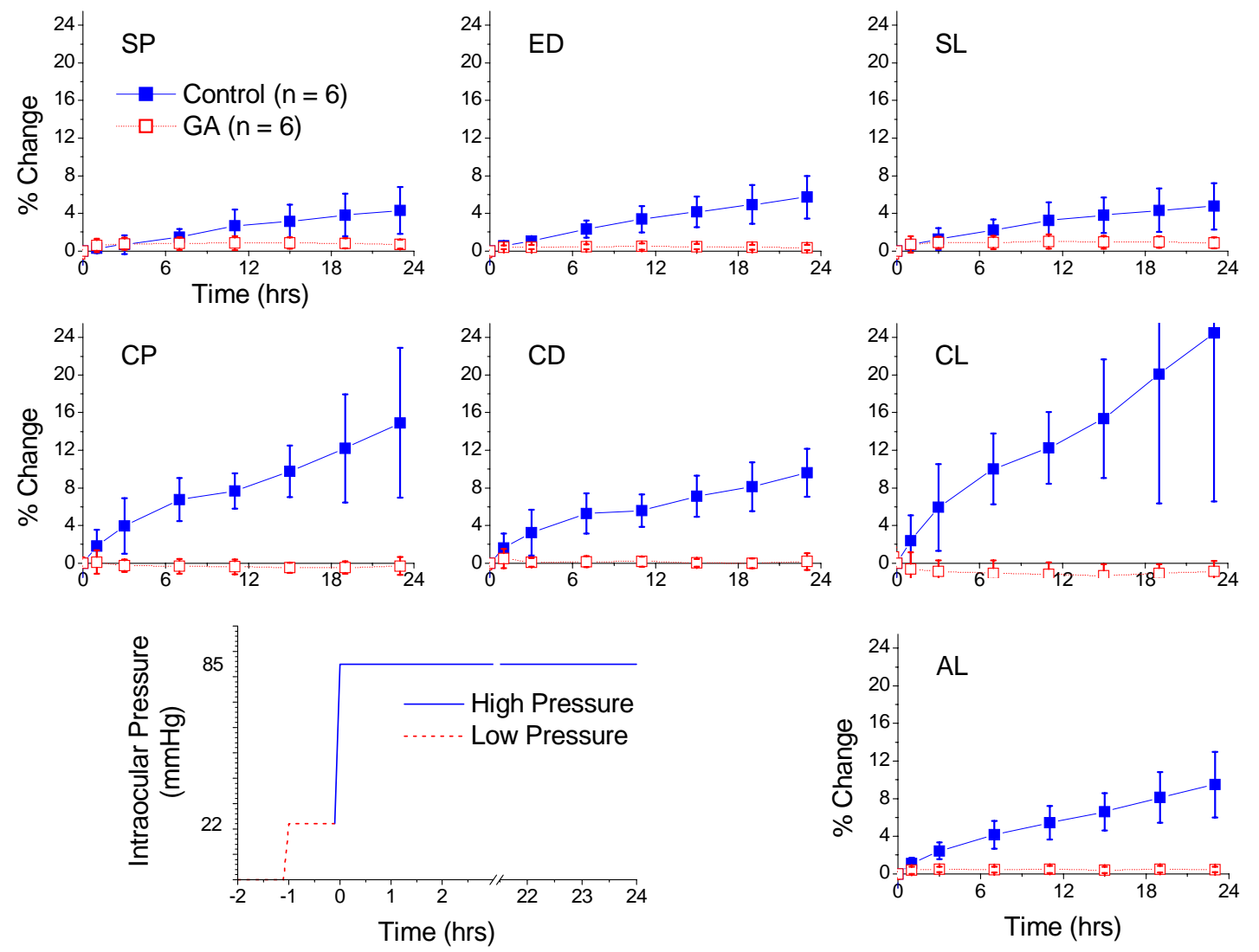

Figure 2.26. Glyceraldehyde (GA) crosslinking of whole eyes stiffens the tissue and noticeably changes the response of the intact eyes to high pressure. While control eyes expand along every dimension, the treated eyes show little expansion along any of the dimensions.

Treatments that prevent expansion of the eyes in vitro show the potential to strengthen the tissue and prevent distension in vivo. Using the intact globe method, we are able to screen potential treatments with relatively few animals as compared to the tensile and shear tests. By using a test that has resemblance to physiological stresses, we are more likely to probe conditions that are relevant to stopping corneal and scleral shape changes in keratoconus and degenerative myopia. For these reasons, the intact globe method has been chosen to demonstrate treatment efficacy in later chapters. 
The expansion studies developed here could also find application in the study of eyes with altered mechanical properties. Currently, we use the tests to induce deformation in normal tissue, but studies of diseased tissue may show increased susceptibility to distension with worsening disease state. While this has been studied with tensile measurements, we believe that this method will provide a fuller characterization of the changes. Studies of molecules such as retinoic acid, which is known to cause increased axial growth in chicks, could provide insight to the mechanisms by which changes occur in the sclera (cell signaling, or direct effects on collagen). Such future work with this model is likely to yield new insights to the field of eye biomechanics. 


\section{BIBLIOGRAPHY}

1. Hoeltzel, D.A., Altman, P., Buzard, K., Choe, K.I. Strip Extensiometry for Comparison of the Mechanical Response of Bovine, Rabbit, and Human Corneas. Journal of Biomechanical Engineering-Transactions of the ASME 114, 202215 (1992).

2. Zeng, Y.J., Yang, J., Huang, K., Lee, Z.H., Lee, X.Y. A comparison of biomechanical properties between human and porcine cornea. Journal of Biomechanics 34, 533-537 (2001).

3. Andreassen, T.T., Simonsen, A.H., Oxlund, H. Biomechanical Properties of Keratoconus and Normal Corneas. Experimental Eye Research 31, 435-441 (1980).

4. Bryant, M.R., Szerenyi, K., Schmotzer, H., Mcdonnell, P.J. Corneal TensileStrength in Fully Healed Radial Keratotomy Wounds. Investigative Ophthalmology \& Visual Science 35, 3022-3031 (1994).

5. Curtin, B.J. Physiopathologic aspects of scleral stress-strain. Trans Am Ophthalmol Soc 67, 417-61 (1969).

6. Downs, J.C., Suh, J.K.F., Thomas, K.A., Bellezza, A.J., Burgoyne, C.F., Hart, R.T. Viscoelastic characterization of peripapillary sclera: Material properties by quadrant in rabbit and monkey eyes. Journal Of Biomechanical EngineeringTransactions Of The ASME 125, 124-131 (2003).

7. Friberg, T.R., Lace, J.W. A Comparison of the Elastic Properties of Human Choroid and Sclera. Experimental Eye Research 47, 429-436 (1988). 
8. McBrien, N.A., Gentle, A. Role of the sclera in the development and pathological complications of myopia. Progress In Retinal And Eye Research 22, 307-338 (2003).

9. Nash, I.S., Greene, P.R., Foster, C.S. Comparison of Mechanical-Properties of Keratoconus and Normal Corneas. Experimental Eye Research 35, 413-424 (1982).

10. Nyquist, G.W. Rheology of Cornea - Experimental Techniques and Results. Experimental Eye Research 7, 183-188 (1968).

11. Pandolfi, A., Manganiello, F. A model for the human cornea: constitutive formulation and numerical analysis. Biomechanics and Modeling in Mechanobiology 5, 237-246 (2006).

12. Phillips, J.R., Khalaj, M., McBrien, N.A. Induced myopia associated with increased scleral creep in chick and tree shrew eyes. Investigative Ophthalmology \& Visual Science 41, 2028-2034 (2000).

13. Phillips, J.R., McBrien, N.A. Form Deprivation Myopia - Elastic Properties Of Sclera. Ophthalmic And Physiological Optics 15, 357-362 (1995).

14. Reichel, E., Miller, D., Blanco, E., Mastanduno, R. The Elastic-Modulus of Central and Perilimbal Bovine Cornea. Annals of Ophthalmology 21, 205-208 (1989).

15. Shchukin, E.D., Izmailova, V.N., Larionova, N.I., Krasnov, M.M., Gurov, A.N., Bessonov, A.I., Afanas'eva, G.N. Creep of the eye sclera. Materials Research Innovations 2, 147-149 (1998).

16. Siegwart, J.T., Norton, T.T. Regulation of the mechanical properties of tree shrew sclera by the visual environment. Vision Research 39, 387-407 (1999). 
17. Spoerl, E., Boehm, A.G., Pillunat, L.E. The influence of various substances on the biomechanical behavior of lamina cribrosa and peripapillary sclera. Investigative Ophthalmology \& Visual Science 46, 1286-1290 (2005).

18. Spoerl, E., Boehm, A.G., Valtink, M., Pillunat, L.E. Changes of biomechanical properties of lamina cribrosa and of peripapillary sclera by glyceraldehyde. Investigative Ophthalmology \& Visual Science 45, U789 (2004).

19. Spoerl, E., Hellmund, K., Schreiber, J., Seiler, T. In vivo-stiffening of the rabbit cornea by riboflavin plus UV-irradiation and glutaraldehyde. Investigative Ophthalmology \& Visual Science 40, S339 (1999).

20. Spoerl, E., Huhle, M., Seiler, T. Induction of cross-links in corneal tissue. Experimental Eye Research 66, 97-103 (1998).

21. Spoerl, E., Seiler, T. Techniques for stiffening the cornea. Journal Of Refractive Surgery 15, 711-713 (1999).

22. Wollensak, G., Spoerl, E. Collagen crosslinking of human and porcine sclera. Journal Of Cataract And Refractive Surgery 30, 689-695 (2004).

23. Wollensak, G., Spoerl, E., Seiler, T. Stress-strain measurements of human and porcine corneas after riboflavin-ultraviolet-A-induced cross-linking. Journal Of Cataract And Refractive Surgery 29, 1780-1785 (2003).

24. Pinsky, P.M., Datye, D.V. A microstructurally-based finite element model of the incised human cornea. Journal of Biomechanics 24, 907-909 (1991).

25. Soergel, F., Muecke, S., Pechhold, W. Corneal Viscoelasticity Spectra as a Result of Dynamic Mechanical Analysis. in Advances in Corneal Research: Selected Transactions of the World Congress on the Cornea IV (ed. J. H. Lass) 257-272 (1997). 
26. Mattson, M., Schwartz, D.M., Kornfield, J.A. Mechanical measurements of sclera for screening myopia treatments. Investigative Ophthalmology \& Visual Science 46(2005).

27. Nickerson, C.S., Karageozian, H.L., Park, J., Kornfield, J.A. The mechanical properties of the vitreous humor. Investigative Ophthalmology \& Visual Science 45, U113 (2004).

28. Nickerson, C.S., Karageozian, H.L., Park, J., Kornfield, J.A. Chemical resilience of the vitreous and vitreoretinal interface. Investigative Ophthalmology \& Visual Science 46, 5165 (2005).

29. Nickerson, C.S., Kornfield, J.A. A "cleat" geometry for suppressing wall slip. Journal Of Rheology 49, 865-874 (2005).

30. Prausnitz, M.R., Noonan, J.S. Permeability of cornea, sclera, and conjunctiva: A literature analysis for drug delivery to the eye. Journal Of Pharmaceutical Sciences 87, 1479-1488 (1998).

31. Jin, M.S., Grodzinsky, A.J. Effect of electrostatic interactions between glycosaminoglycans on the shear stiffness of cartilage: A molecular model and experiments. Macromolecules 34, 8330-8339 (2001).

32. Kuettner, K.K.E., Lindenbaum, A.A. Analysis of mucopolysaccharides in partially aqueous media. Biochimica et biophysica acta 101, 223-5 (1965).

33. Nishiyama, N., Suzuki, K., Nagatsuka, A., Yokota, I., Nemoto, K. Dissociation States of Collagen Functional Groups and their Effects on the Priming Efficacy of HEMA Bonded to Collagen. J Dent Res 82, 257-261 (2003).

34. Greene, P.R., Mcmahon, T.A. Scleral Creep Vs Temperature and Pressure Invitro. Experimental Eye Research 29, 527-537 (1979). 
35. Arciniegas, A., Amaya, L.E. Mechanical-Behavior of the Sclera. Ophthalmologica 193, 45-55 (1986).

36. Cahane, M., Bartov, E. Axial Length and Scleral Thickness Effect on Susceptibility to Glaucomatous Damage - a Theoretical-Model Implementing Laplaces Law. Ophthalmic Research 24, 280-284 (1992).

37. Forster, W., Kasprzak, H., Vonbally, G. Measurement of Elastic-Modulus of the Central Bovine Cornea by Means of Holographic-Interferometry .2. Results. Optometry and Vision Science 71, 27-32 (1994).

38. Girard, M., Downs, J.C., Burgoyne, C.F., Suh, J.K.F. Experimental surface strain mapping of porcine peripapillary sclera under Intraocular pressure. Investigative Ophthalmology \& Visual Science 46, 1271 (2005).

39. Greene, P.R. Mechanical Considerations in Myopia - Relative Effects of Accommodation, Convergence, Intraocular-Pressure, and the Extra-Ocular Muscles. American Journal of Optometry and Physiological Optics 57, $902-914$ (1980).

40. McEwen, W.K., Sthelen, R. Rheology Of Human Sclera - Unifying Formulation Of Ocular Rigidity. Ophthalmologica 150, 321-346 (1965).

41. Phillips, J.R., McBrien, N.A. Pressure-induced changes in axial eye length of chick and tree shrew: Significance of myofibroblasts in the sclera. Investigative Ophthalmology \& Visual Science 45, 758-763 (2004).

42. Pierscionek, B.K., Widlicka, M., Schachar, R.A. The effect of changing intraocular pressure on the corneal and scleral curvatures in the fresh porcine eye. Br J Ophthalmol, bjo.2006.110221 (2006). 
43. Purslow, P.P., Karwatowski, W.S.S. Ocular elasticity - Is engineering stiffness a more useful characterization parameter than ocular rigidity? Ophthalmology 103, 1686-1692 (1996).

44. Richards, R.D., Tittel, P.G. Corneal and Scleral Distensibility Ratio on Enucleated Human Eyes. Investigative Ophthalmology 12, 145-151 (1973).

45. Sampson, W.G., Girard, L.J. The coefficient of scleral rigidity. Effect of variation of the intraocular volume. Am J Ophthalmol 52, 789-99 (1961).

46. Sthelen, R., McEwen, W.K. Rheology Of Human Sclera .1. Anelastic Behavior. American Journal Of Ophthalmology 52, 539-548 (1961).

47. Sthelen, R., McEwen, W.K. Rheology Of Human Sclera. American Journal Of Ophthalmology 51, 328 (1961).

48. Tittel, P.G., Richards, R.D. Distensibility Measurements of Rabbit Eye. Investigative Ophthalmology 10, 800-809 (1971).

49. Woo, S.L.Y., Schlegel, W.A., Kobayash, A.S., Lawrence, C. Nonlinear Material Properties of Intact Cornea and Sclera. Experimental Eye Research 14, 29-39 (1972). 